\title{
Orally Bioavailable and Effective Buparvaquone Lipid Based Nanomedicines for Visceral Leishmaniasis
}

Lindsay Smith ${ }^{a}$, Dolores R. Serrano ${ }^{b}$, Marion Mauger ${ }^{a}$, Francisco Bolás-Fernández ${ }^{c}$, Maria Auxiliadora Dea-Ayuela ${ }^{d}$, Aikaterini. Lalatsa ${ }^{*}$

Institute of Biomedical and Biomolecular Sciences, School of Pharmacy and Biomedical Sciences, University of Portsmouth, White Swan Road, Portsmouth, PO1 2DT, UK.

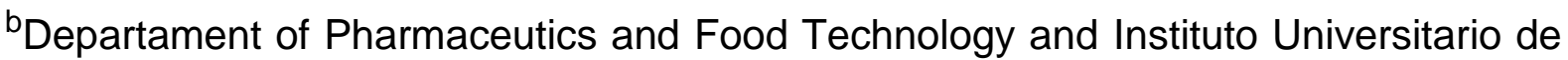
Farmacia Industrial (IUFI), School of Pharmacy, University Complutense, Avenida Complutense, 28040 Madrid, Spain.

'Departament of Microbiology and Parasitology, School of Pharmacy, Universidad Complutense de Madrid, Plaza Ramón y Cajal s/n, 28040-Madrid, Spain.

dDepartamento de Farmacia, Facultad de Ciencias de la Salud, Universidad CEU Cardenal Herrera, Edificio Seminario s/n, 46113-Moncada, Valencia, Spain.

\section{*Author for correspondence}

Dr. Aikaterini Lalatsa, Senior Lecturer in Pharmaceutics, Institute of Biomedical and Biomolecular Sciences, School of Pharmacy and Biomedical Sciences, University of Portsmouth, White Swan Road, Portsmouth, PO1 2DT, UK, Email: katerina.lalatsa@port.ac.uk, Tel: +44 2392843929 


\section{Graphical Abstract}
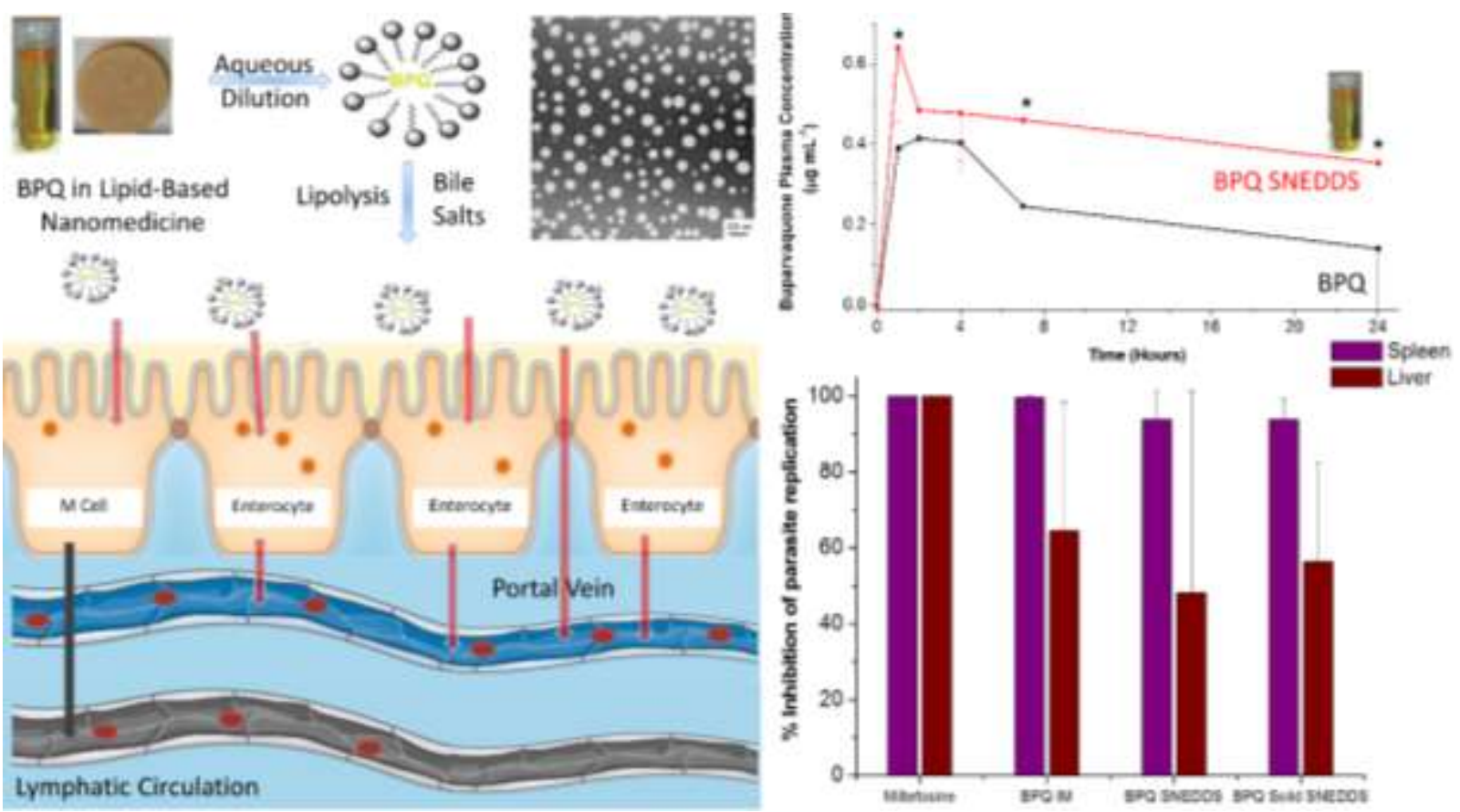


\begin{abstract}
Nano-enabled lipid based drug delivery systems offer a platform to overcome challenges encountered with current failed leads in the treatment of parasitic and infectious diseases. When prepared with FDA or EMA approved excipients, they can be readily translated without the need for further toxicological studies, while they remain affordable and amenable to scale-up. Buparvaquone (BPQ), a hydroxynapthoquinone with in vitro activity in the nanomolar range, failed to clinically translate as a viable treatment for visceral leishmaniasis due to its poor oral bioavailability limited by its poor aqueous solubility (BCS Class II drug). Here we describe a self-nanoemulsifying system (SNEDDS) with high loading and thermal stability up to 6 months in tropical conditions able to enhance the solubilisation capacity of BPQ in gastrointestinal media as demonstrated by flow-through cell and dynamic in vitro lipolysis studies. BPQ SNEDDS demonstrated an enhanced oral bioavailbility compared to aqueous BPQ dispersions (probe - sonicated) resulting in an increased plasma $\mathrm{AUC}_{0-24}$ by $55 \%$ that is four fold higher than any previous reported values for BPQ formulations. BPQ SNEDDS can be adsorbed on low molecular glycol chitosan polymers forming solid dispersions that when compressed into tablets allow the complete dissolution of BPQ in gastrointestinal media. BPQ SNEDDS and BPQ solid SNEDDS demonstrated potent in vitro efficacy in the nanomolar range $(<37 \mathrm{nM})$ and were able to near completely inhibit parasite replication in the spleen and $48 \pm 48$ and $56 \pm 23 \%$ inhibition of the parasite replication in the liver respectively compared to oral miltefosine after daily administration over 10 days. The proposed platform technology can be used to elicit a range of cost-effective and orally bioavailable non-invasive formulations for a range of antiparasitic and infectious disease drugs that are needed for closing the global health innovation gap.
\end{abstract}

\title{
Key Words
}

Neglected parasitic diseases, Visceral Leishmaniasis, Buparvaquone, Oral Delivery, Self-nanoemulsifying drug delivery systems (SNEDDS), Solid Nanomedicines. 


\section{Introduction}

Advances in biotechnology and drug delivery have transformed medicine in the industrialised world, but these innovations have yet to reach developing countries. More than 10 million people in the developing countries die of infectious and parasitic diseases such as malaria, leishmaniasis, HIV/AIDS, tuberculosis, and diarrheal diseases annually, while morbidity related to these diseases devastates economic development ${ }^{1}$. More importantly, the burden of infectious and parasitic illness falls more heavily on children and pregnant women ${ }^{2}$.

Many of these diseases have earned the label of "neglected", because health-care markets in the affected countries are insufficient to attract pharmaceutical industry funding in research and development. These involve African Trypanosoniasis, Chagas diseases, dengue, leishmaniasis, leprosy, lymphatic filariasis, malaria, onchocerciasis, schistosomiasis and tuberculosis ${ }^{3}$. Over the past decade, publicprivate, not-for-profit, product development partnerships (PDPs) have arisen to tackle the development of new drugs, vaccines, diagnostics for developing-world diseases, while governments and foundations have increased their investment in neglected diseases research and development. The challenge remains to augment these public and private sector efforts by providing to those partnerships access to advanced technologies and expertise for discovering, delivering and developing new medicines 4.

Leishmaniasis presents as cutaneous, mucosal and visceral forms caused by protozoa of the genus Leishmania that spread in humans through the bite of a phlebotomine sandfly ${ }^{5}$. The cutaneous and mucosal forms cause severe disfigurement and disability resulting in gender inequality, stigmatisation and reduction of economic productivity ${ }^{6}$, while the visceral $(V L)$ form is fatal if left untreated (with a 
$100 \%$ fatality rate within 2 years). An estimated 12 million people are infected with Leishmania with 50,000 deaths annually ${ }^{7}$, while 1.3 million new cases are reported every year putting in risk 310 million people ${ }^{6}$ over a wider geographical distribution today due to urbanisation, migration and deforestation, making leishmaniasis a growing public health concern ${ }^{8}$. To complicate things further, up to twelve percent of all VL cases are associated with HIV/AIDS, while highest prevalence is reported in sub-Saharan countries $(20-40 \% \text { of cases })^{9-11}$.

First line treatment for VL relies on pentavalent antimonials [sodium stibogluconate (SSG) and $\mathrm{N}$-methylglucamine antimoniate (meglumine antimoniate) ${ }^{12}$. As resistance to antimonials is increasing limiting their use, emerging second-line treatments involve pentamidine and Amphotericin $B(A m B)^{13}, 14 . A m B$ is highly effective in VL when administered intravenously. Available parenteral formulations involve an affordable micellar formulation (Fungizone ${ }^{\circledR}$ ), which is highly nephrotoxic limiting the dose able to be administered, and a liposomal formulation $\left(\right.$ Ambisome $\left.^{\circledR}\right)$ with the latter used first line in the developed world. Other parenteral formulations involve lipidic complexes $\left(\right.$ Abelcet $\left.^{\circledR}\right)$, but these are less likely to be used due to their higher cost compared to Ambisome $^{\circledR 13}$. As AmB parenteral formulations are thermally unstable, cost of therapy is raised further to account for the need for cold transport and refrigeration. To overcome this issue, the clinical use of a heated Fungizone ${ }^{\circledR}$ has been attempted in in India. This involved a 10 -fold aqueous dilution of Fungizone ${ }^{\circledR}$ and heating to $70{ }^{\circ} \mathrm{C}$ for 20 minutes ${ }^{15}$, which elicits larger AmB aggregates that are not renally cleared reducing observed nephrotoxicity ${ }^{16}$. Pentamidine, on the other hand, has been shown to cause insulin dependent diabetes which is irreversible and potentially fatal ${ }^{17}$. Miltefosine, the only oral VL licensed treatment (licensed in 2003 in India), has a long elimination half-life (7 days) and a narrow therapeutic index. These pharmacokinetic 
characteristics limit the administered dose which can result in subtherapeutic levels over several weeks encouraging the emergence of resistance ${ }^{18}$. Indeed, resistance has been reported in India (a county that alone accounts for $50 \%$ of the VL worldwide burden) and France ${ }^{13,19}$. Thus, available medicines for VL are outdated, impractical (non-oral, requiring hospitalisation and access to healthcare professionals over long treatment periods), insufficiently efficacious, or subject to resistance and unacceptable toxicities ${ }^{4,20-22}$. Based on this, an ideal treatment for VL would possess greater than $95 \%$ efficacy, be orally administered, be stable in a tropical environment, be affordable and require less than 11 days treatment with an excellent side-effect profile ${ }^{23}$. Buparvaquone (BPQ), a hydroxynapthoquinone licensed for theileriosis as an intramuscular injection, possesses promising activity in the nanomolar range against Leishmania spp. amastigotes (ED50: $0.005 \mu \mathrm{M}$ against $L$. donovani) ${ }^{24}$. Although in vitro experiments have provided promising results in the 90 s, in vivo activity up to oral doses of $100 \mathrm{mg} \mathrm{kg}^{-1}$ has been low, due to its poor solubility in physiological fluids (<300 ng $\mathrm{mL}^{-1}, \log \mathrm{D}: 7.02$ at $\mathrm{pH} 3.0$ ) and low oral bioavailability 24,25 , which hindered its translation into a viable therapy. Attempts to improve BPQ's aqueous solubility and bioavailability have included the generation of water soluble BPQ phosphate prodrugs 26-28, the use of nebulised nanosuspensions 29 BPQ nanosuspensions in mucoadhesive hydrogels ${ }^{30}$, oral suspensions and cyclodextrin complexes with limited success $^{31}$. The most promising results towards an oral bioavailable BPQ formulations involved the use of self-emulsifying drug delivery system (SEDDS) ${ }^{25}$. However, the oral efficacy of the BPQ SEDDS have not been demonstrated. Here we present, a novel BPQ loaded self-nanoemulsifying drug delivery system (BPQ-SNEDDS) with potent in vitro and in vivo activity along with oral pharmacokinetic and pharmacodynamic studies. Additionally, we present a facile easily scalable method to 
convert these BPQ-SNEDDS into solid nanomedicines for the treatment of VL. The proposed formulations are cost-effective, thermally stable and prepared from generally regarded as safe excipients (GRAS) that can enable their facile clinical translation, while they can be used for other poorly antiparasitic and chemotherapeutic drugs for the treatment of parasitic and infectious diseases.

\section{Methods}

\section{Materials}

Buparvaquone (>95\% HPLC) was obtained from Toronto Research Chemicals (Toronto, Canada). Capryol 90 (propylene glycol monocaprylate 90\%), Labrasol (Labrasol ALF, Caprylocaproyl macragol-8 glycerides), Labrafil M 1944 CS (Oleoylocapryoyl macragol-6 glycerides) were kindly provided by Gattefosse (Berkshire, UK) and soya phosphatidylcholine (Lipoid S100) by Lipoid GmbH (Rein, Germany). Pancreatin (porcine, P1625, $\geq 3$ USP), bile extract (porcine) and all other chemicals, solvents, and acids, unless otherwise stated, were of ACS grade or above and were obtained from Sigma-Aldrich (Dorset, UK) and used without further purification. Cell culture media were bought from Sigma-Aldrich (Madrid, Spain).

\section{Preparation of BPQ loaded SNEDDS (BPQ SNEDDS)}

BPQ loaded SNEDDS (BPQ SNEDDS) were prepared by combining BPQ, Labrafil 1944CS (oleoyl macragol-6 glycerides), Capryol 90 (propylene glycol monocaprylate) and Labrasol (caprylocaproyl macragol-8 glycerides) (0.01:3:1:5.99 w/w) under stirring at $600 \mathrm{rpm}$. This ratio was identified using pseudo-ternary phase diagram studies constructed with varying concentrations of oily solubilisers (Labrafil M 1944 CS) and oil soluble surfactants (Labrasol) and co-surfactants (Capryol 90) and double-distilled water using the water titration method at room temperature ${ }^{25}$ (Figure SI-1). The 
samples were placed into a water bath at $37 \pm 0.5^{\circ} \mathrm{C}$ for 24 hours under gentle shaking (Kotterman D1365, Hänigsen, Germany). Mixtures were then observed for turbidity when fresh and at 24 hours and particle size was measured when fresh and after 24 hours as described below. Turbidity is indicative of the formation of a coarse emulsion, whereas a clear isotropic solution would indicate a microemulsion (type II).

All formulations containing BPQ were protected from light. BPQ SNEDDS (10, 20 and $50 \mathrm{mg} \mathrm{mL}^{-1}$ ) were prepared in triplicate as described above to determine the maximum $\mathrm{BPQ}$ loading within the SNEDDS. After $24 \mathrm{~h}$, the samples were taken out from the waterbath and centrifuged at 10,000 rpm for 10 minutes (Jouan B4i centrifuge, Thermo Scientific, USA) and the supernatant was isolated and analysed using HPLC (after a 1 in 250 dilution with methanol) as described below.

\section{Photon Correlation Spectroscopy (particle size \& zeta potential measurements)}

SNEDDS were diluted with water at 1 in $200 \mathrm{v} / \mathrm{v}$ and 1 in $100 \mathrm{v} / \mathrm{v}(\mathrm{pH} \mathrm{6.5)}$ prior to particle size and zeta-potential measurements respectively (Malvern Zetasizer NanoZs, Malvern Instruments, Worcestershire, UK) at $25^{\circ} \mathrm{C}$ and at a wavelength of 633 nm. The data were analysed using the Contin method of data analysis. Diluted suspensions were left for 15 minutes at room temperature $\left(25^{\circ} \mathrm{C}\right)$ before particle size was measured. The accuracy of the instrument was assessed periodically using a drop of latex beads (polystyrene, mean size: $0.1 \mu \mathrm{m}$ ) in $50 \mathrm{mM}$ sodium chloride (dispersed phase). All measurements $(n=13)$ were performed in triplicate and the mean $( \pm S D)$ was reported.

Zeta potential (Malvern Nano-Zs, Malvern Instruments, UK) was measured for the diluted formulations utilizing the Doppler electrophoresis technique. Analysis of the Doppler shift (Fourier transformed) was done by using a mixed-mode measurement phase analysis light scattering (M3-Pals). The viscosity of the sample was 
hypothesized to be the viscosity of water at $25^{\circ} \mathrm{C}$. All measurements $(n=100)$ were performed in triplicate and the mean $( \pm S D)$ was reported.

\section{HPLC analysis}

A modified reverse phase HPLC (RP-HPLC-UV) method based on the optimized and validated method published by Venkatesh et al ${ }^{32}$ was used. A Phenomenex Onyx Monolithic $\mathrm{C} 18$ column $(10+100 \mathrm{~mm} \times 4.6 \mathrm{~mm}, 5 \mu \mathrm{m})$ was maintained at $30^{\circ} \mathrm{C}$ (Varian Prostar column heater) for analysis and the analytical wavelength was set to $251 \mathrm{~nm}$ and 342 nm (Varian Prostar, Agilent technologies Inc, USA). The mobile phase consisted of $1 \%$ acetic acid in water, acetonitrile and methanol in the ratio $30: 60: 10$ $(\mathrm{v} / \mathrm{v} / \mathrm{v})$ and was filtered (Millipore nylon filter $0.45 \mu \mathrm{m})$ and degassed using bath sonication (175W, Jencons scientific Ltd, Bedfordshire, UK). The injection volume was set at $40 \mu \mathrm{L}$ per sample using Varian Prostar autosampler (model 410, Agilent technologies Inc, USA) and a flow rate of $1.5 \mathrm{~mL} \mathrm{~min}{ }^{-1}$ was achieved using a Varian Prostar solvent delivery module (Agilent technologies Inc, USA). The retention time was 5.54 minutes for $B P Q$ and the lowest detection limit was set at $0.05 \mu \mathrm{gL}^{-1}$. A linear calibration curve in a range of 0.05 to $100 \mathrm{\mu g} \mathrm{mL}^{-1}$ was used for extrapolation of the concentrations.

\section{Flow-through cell dissolution studies for BPQ SNEDDS}

The dissolution of BPQ from BPQ-SNEDDs (10 $\mathrm{mg} \mathrm{mL}^{-1}, 0.3 \mathrm{~mL}$ ) loaded capsules (NP CapsTM, pullulan, size 2, Capsugel) was studied in the flow through cell (USP, Apparatus IV) at various pH levels [USP 2013 simulated gastric fluid without enzyme (1.2), acetate buffer (4.5) and phosphate buffer (6.8), flow rate $6 \mathrm{ml} \mathrm{min}^{-1}$ ] as previously described ${ }^{14}$.

\section{In vitro lipolysis}


In vitro lipolysis experiments were performed as previously described ${ }^{33}$. The lipolysis medium contained bile salts $(5 \mathrm{mM})$ and phosphatidylcholine $(1 \mathrm{mM})$ which simulate fasted conditions in the gastrointestinal tract (GIT), while the digestion buffer $(300 \mathrm{~mL})$ was composed of sodium chloride $(150 \mathrm{mM})$ and Trizma maleate $(2 \mathrm{mM})$ at $\mathrm{pH} 6.5 \pm$ $0.05^{33}$. A low buffer concentration was chosen to ensure that ionized fatty acids are able to change $\mathrm{pH}$, while a buffer with low capacity was selected to allow the released fatty acids (FA) to reduce the $\mathrm{pH}$ and thus trigger the addition of sodium hydroxide ${ }^{33}$. The lipase suspension was prepared by suspending pancreatin $(16.6 \mathrm{~g})$ in $110 \mathrm{~mL}$ of Millipore water at $37^{\circ} \mathrm{C}$ and thoroughly mixing prior centrifugation $(4,000 \mathrm{rpm}, 7$ minutes at $37^{\circ} \mathrm{C}$ ). The $\mathrm{pH}$ of the supernatant was adjusted to 6.5 using $1 \mathrm{M}$ sodium hydroxide and $100 \mathrm{~mL}$ was used to initiate the lipolysis. To avoid denaturation, preparation of the pancreatin suspension never exceeded 15 minutes. For the in vitro digestion study, the lipolysis medium $(300 \mathrm{~mL})$ were pre-warmed at $37^{\circ} \mathrm{C}$, prior to the addition of BPQ loaded SNEDDS (3g) and adjusting the $\mathrm{pH}$ to $6.5 \pm 0.01$ with $1 \mathrm{M}$ sodium hydroxide. Lipolysis was initiated by adding the $100 \mathrm{~mL}$ of the freshly prepared lipase suspension. $\mathrm{Ca}^{2+}$ solution $(0.5 \mathrm{M})$ was added at a dispensing rate of $0.045 \mathrm{mM}$ $(90 \mu \mathrm{L})$ per min (NE-1000 Programmable single syringe pumps, World Precision Instruments Ltd., Hertfordshire, UK). Throughout the study, the pH was kept constant at $6.5 \pm 0.01$ (Accumet $\mathrm{AB} 200$ equipped with an accuTupH rugged bulb, Fisher Scientific, Loughborough, UK) by addition of $1 \mathrm{M}$ sodium hydroxide. In this respect, the number of $\mathrm{OH}^{-}$ions present in the volume of the titrant can be equated with the $\mathrm{FA}$ liberation caused by lipolysis. Samples $(30 \mathrm{~mL})$ were withdrawn at time $0,5,15,30$, 60 , and 90 minutes after the addition of the lipase suspension. Samples were frozen using dry ice prior ultracentrifugation at $40,000 \mathrm{rpm}$ at $4^{\circ} \mathrm{C}$ (CP100NX, Hitachi Koki Co. Ltd, Milton Keynes, UK). The amount of BPQ present in the supernatant and in 
the pellet was quantified after their appropriate dilution with methanol using HPLC as described above.

\section{Stability studies of BPQ SNEDDS}

Blank SNEDDS and BPQ SNEDDS (10 $\left.\mathrm{mg} \mathrm{mL}^{-1}, 0.5 \mathrm{~mL}, \mathrm{n}=3\right)$ were divided into amber HPLC vials and stored under $75 \%$ relative humidity in amber dessicant jars containing a saturated sodium chloride solution $(20 \mathrm{~g}$ of sodium chloride in $10 \mathrm{~mL}$ of de-ionised water) at $40^{\circ} \mathrm{C}$ (Raven Incubator, LTE Scientific, Oldham, UK). Samples (10 $\left.\mu \mathrm{L}\right)$ were diluted with water (1 in $200 \mathrm{v} / \mathrm{v}, \mathrm{pH}$ 6.5) and analysed for particle size and zetapotential, while samples $(10 \mu \mathrm{g})$ were diluted with methanol and analysed by HPLC as described above at weeks $0,2,3,4,6,10,12,16,20,24$. Morphology of the diluted aqueous dispersions of the stability samples was assessed by transmission electron microscopy at week 0 and week 6 as described below.

\section{Preparation of BPQ loaded solid SNEDDS (BPQ solid SNEDDS)}

Low molecular weight glycol chitosan polymers were produced from $2 \mathrm{~g}$ of glycol chitosan that were added to freshly prepared $4 \mathrm{M}$ hydrochloric acid (152 $\mathrm{mL})$, and stirred (500 rpm, IKA Werke RTC basic, IKA laboratories, Staufen, Germany) until homogenous (25 $\mathrm{min}$ ) as previously described ${ }^{34}$. The solution was then placed in a $50^{\circ} \mathrm{C}$ waterbath under vigorous shaking $(50 \mathrm{rpm})$ for a total of 24 hours (Kotterman D1365, Hänigsen, Germany). The polymer solution was dialysed with six changes (5 L) of de-ionised water over 24 hours (Dialysis membrane Molecular weight cut-off for Cytochrome C: 12-14 kDa, Medicell Ltd, London, UK). The recovered degraded glycol chitosan (GC 14 kDa) was snap-frozen using liquid nitrogen and lyophilised (Edwards Modulyo freeze drier [Crawley, UK] connected to CIT Alcatel vacuum pump [Pfeiffer, Asslar, Germany]) over 72 hours at $-50^{\circ} \mathrm{C}$. 
Blank SNEDDS (3mL) and BPQ SNEDDS (3mL, $\left.10 \mathrm{mg} \mathrm{mL}^{-1}\right)$ were adsorbed onto 400 $\mathrm{mg}$ of degraded glycol chitosan (14 kDa). Croscarmellose sodium (295 mg) was added followed by the addition of lactose BP (5.2 g, BP: British Pharmacopoeia) and mixed in a mortar and pestle. The contents of the mortar were transferred into a roundbottom flask and $60-70 \mathrm{mls}$ of de-ionised water were added and stirred until a homogenous viscous yellow suspension was prepared. The resulting suspension was snap-frozen with liquid nitrogen and lyophilised (Edwards Modulyo freeze drier [Crawley, UK] connected to CIT Alcatel vacuum pump [Pfeiffer, Asslar, Germany]) over 24 hours at $-50^{\circ} \mathrm{C}$. The resulting lyophilised product $(15 \mathrm{mg})$ was diluted with 15 $\mathrm{mL}$ methanol, vortexed for 1 minute (Topmix vortex, Fischer Scientific, Leicestershire, UK), and centrifuged at 10,000 rpm (Jouan B4i centrifuge, Thermo Scientific, USA) for 10 minutes before the supernatant was analysed using HPLC for drug loading as described below. The solid powders were also characterised using DSC, TGA, PXRD, FT-IR, SEM, and particle size as described below. Physical mixtures of lyophilised BPQ and blank solid SNEDDS (1:10 and 1:100 w/w) were also analysed via DSC and PXRD.

\section{Morphological characterisation}

A drop of the diluted SNEDDS or Solid SNEDDS (1 in $200 \mathrm{v} / \mathrm{v}$ or 1 in $200 \mathrm{w} / \mathrm{v}$ ) was placed on Formvar®/Carbon Coated Grid (F196/100 3.05 mm, Mesh 300, Tab Labs Ltd, England) and stained with 1\% aqueous Uranyl Acetate before TEM analysis as previously described (FEI CM120 BioTwin Transmission Electron Microscope) ${ }^{35}$.

Dry powders left to air dry was mounted on a glass slide that was mounted on a standard SEM sample holder and fixed on a brass/aluminium stub using double sided carbon impregnated adhesive discs. The sample was then sputtered coated with a conducting gold-palladium (10 nm, 60\% gold-palladium) coating using a SEM coating 
system for 2 minutes at 30 mA (Quorum Q 15ORES, Quorum technologies Ltd, Lewes, UK, Deposition range: 0-80 mA, Deposition rate: 0- $25 \mathrm{~nm} \mathrm{~min}^{-1}$, Sputter timer: 0-60 min, Vacuum Pump: $50 \mathrm{~L} \mathrm{~min}^{-1}$, room temperature) before being viewed and photographed under a range of magnifications under high vacuum using JEOL JSM6060LV (Jeol, Welwyn Garden City, UK) scanning electron microscope.

\section{Powder X-Ray Diffraction (PXRD)}

PXRD analysis was performed with a Rigaku Miniflexll desktop X-ray diffractometer (Rigaku, Tokyo, Japan) attached to a Haskris cooling unit (Grove Village, IL, USA). The PXRD patterns were recorded from $5^{\circ}$ to $40^{\circ}$ on the 2 theta at a step scan rate of $0.05^{\circ}$ per second as previously described $(n=3)^{36}$. X-ray powder patterns of single crystal structures were calculated using Mercury® (version 3.9., Cambridge Crystallographic Data Centre, Cambridge, UK) and Miller's indices of various facets of the crystal were identified $(\mathrm{SI}-3)^{37}$. Mercury ${ }^{\circledR}$ was also used to predict the Bravais, Friedel, Donnay and Harker (BFDH) morphology to provide an insight into the molecular arrangement of different crystal facets of BPQ crystals (SI-3) ${ }^{37}$.

\section{Thermal analysis}

Differential Scanning Calorimetry (DSC) experiments were performed using a Mettler Toledo DSC 821e (Greifensee, Switzerland) with a refrigerated cooling system (LabPlant RP-100, Filey, UK) as previously described ${ }^{36}$ with nitrogen as the purge gas. Aluminum sample holders $(40 \mu \mathrm{L})$ were sealed with a lid and pierced to provide three vent holes. Sample weight (3-6 mg) was sufficient to provide proper contact between the powder and the bottom of the pan. DSC measurements were carried out at a heating/cooling rate of $10^{\circ} \mathrm{C} \mathrm{min}^{-1}$. The unit was calibrated with indium standards. The DSC system was controlled by Mettler Toledo STARe software (version 6.10) 
working on a Windows NT operating system. Temperatures of melting and crystallisation events refer to onset temperatures. Details for thermogravimetric analysis (TGA) is provided in supplementary information (SI-4).

\section{Fourier-Transformed Infra-red (FT-IR) spectroscopy}

The infrared absorption spectra were obtained using a PerkinElmer Spectrum 1 FTIR Spectrometer (PerkinElmer, Massachusetts, USA) equipped with a Universal Attenuated Total Reflectance (UATR) accessory and a diamond/zinc selenide (4000 to $650 \mathrm{~cm}^{-1}$ ) crystal accessory ${ }^{36}$. Baseline correction and data normalization were performed using Spekwin32 version 1.71.6.1 (maximum resolution is $1 \mathrm{~cm}^{-1}$ ).

\section{Hausner ratio and compressibility index of BPQ solid SNEDDS}

BPQ solid SNEDDS (1 g) was placed in a $5 \mathrm{~mL}$ measuring cylinder and the volume was recorded. Tapped density was calculated by tapping cylinder into a hard surface $10,20,40,60,80,100,120$, and 150 times, recording the volume after each set of taps. The Hausner ratio and compressibility index were determined as described by the equations below:

Compressibility Index $=100 \frac{\mathrm{V} o-\mathrm{V} f}{\mathrm{~V} o}$

Hausner Ratio $=\frac{\mathrm{V} o}{\mathrm{~V} f}$

where $\mathrm{Vo}$ is the unsettled apparent volume and $\mathrm{V} f$ is the final tapped volume.

\section{Preparation and characterisation of BPQ solid SNEDDS tablets}

Lyophilised BPQ solid SNEDDs (300 mg) or blank solid SNEDDS (300 mg) were compressed for 10 seconds under 1 bar of pressure using a flat-faced $12 \mathrm{~mm}$ die (Specac manually operated hydraulic press, Analytical accessories Ltd, Kent, UK). 
Disintegration was tested as specified in the British Pharmacopoeia $2018^{38}$. Tablets were placed in a basket rack assembly, and raised/lowered 30 times per minute at a distance of $55 \pm 2 \mathrm{~mm}$ in $1 \mathrm{~L}$ of water heated to $37.0 \pm 0.5^{\circ} \mathrm{C}$ (Erweka disintegration tester ZT500 series, Erweka GmBH, Heusenstamm, Germany).

Friability studies were conducted using 10 BPQ solid SNEDDS tablets $(300 \mathrm{mg})$ according to British Pharmacopoeia 2018 method for uncoated tablets. Friability was expressed as the percentage of weight loss that was calculated by weighing the tablets after 100 rotations of the drum in $4 \mathrm{~min}$. Tablets were dusted and accurately weighted. Six BPQ solid SNEDDS tablets (300 mg) were crushed using a mortar and pestle and the powder was extracted with $50 \mathrm{~mL}$ of methanol, filtered using Whatman No 1 filter paper and the filtrate was made up to $100 \mathrm{~mL}$ with methanol in a volumetric flask. The concentration in the final solution was measured using HPLC as described above to identify the uniformity of content. The dissolution of six BPQ solid SNEDDS tablets (300 mg) were tested using the basket method (Appendix I, BP 2018) using a GB Caleva Ltd Ascott model 657 dissolution apparatus, with a Tecam TE7 Tempette heating system. Tablets were immersed in $500 \mathrm{~mL}$ of $\mathrm{pH} 1.2 \pm 0.1$ for the first hour, after which $400 \mathrm{~mL}$ of $\mathrm{pH} 6.8$ buffer were added and the final $\mathrm{pH}$ was adjusted to 6.8 using $5 \mathrm{M}$ sodium hydroxide (between $4-4.5 \mathrm{~mL}$ ). The first media was prepared by mixing sodium chloride $(2 \mathrm{M}, 250 \mathrm{~mL})$ and hydrochloric acid $(2 \mathrm{M}, 425 \mathrm{~mL})$ and making them up to $1 \mathrm{~L}$ with deionised water $(\mathrm{pH} 1.2)$. The second media contained $250 \mathrm{~mL}$ potassium dihydrogen phosphate $(2 \mathrm{M}, 250 \mathrm{~mL})$ and sodium hydroxide $(2 \mathrm{M}, 112 \mathrm{~mL})$ that were made up to $1 \mathrm{~L}$ with deionised water $(\mathrm{pH} 6.8)$. Samples $(5 \mathrm{~mL})$ were removed at $2,4,8,10,15,20,25,30,40,50,60,75,90,105,120,145,150,165$ and 180 minutes and replaced with appropriate volume of the required buffer. Samples were then diluted 1:1 with methanol, vortexed for 30 seconds (Topmix vortex, Fischer 
Scientific, Leicestershire, UK), centrifuged at 10,000 rpm for 10 minutes (Jouan B4i centrifuge, Thermo Scientific, USA) before BPQ concentration was quantified by HPLC.

\section{In vitro leishmanicidal assays}

L. Infantum (MCAN/ES/92/BCN83) obtained from an asymptomatic dog from the Priorat region (Catalonia, Spain) were kindly donated by Prof Montserrat Portus (Universidad de Barcelona) ${ }^{39}$. Promastigotes were cultured in Schneider's Insect Medium at $26^{\circ} \mathrm{C}$ supplemented with $20 \%$ heat-inactivated foetal bovine serum (FBS) and $100 \mathrm{U} \mathrm{mL}^{-1}$ of penicillin plus $100 \mu \mathrm{gL}^{-1}$ of streptomycin ${ }^{39}$.

Promastigote drug susceptibility was determined by using the sensitive fluorimetric resazurin model (Sigma-Aldrich ${ }^{\circledR}$ ) as previously described by our group ${ }^{40}$. Briefly, cultured log phase promastigotes $\left(2.5 \times 10^{5}\right.$ parasites per well) were plated in 96 -well flat-bottomed microtiter plates $(200 \mu \mathrm{L} /$ well final volume), in the presence of different concentrations of the studied drug or formulations. Finally, the relative fluorescence units (Excitation: $535 \mathrm{~nm}$, Emission: $590 \mathrm{~nm}$ ) was measured (Infinite 200, Tecan iControl, Männedorf, Switzerland) to determine the growth inhibition rate and then the $\mathrm{IC}_{50}$ calculated by Probit analysis and the statistic parameters were computed using SPSS Statistics v17.0 Software.

Amastigote assays were performed using a fluorimetric assay based on resazurin validated by our group ${ }^{41}$. Macrophages were plated $\left(5 \times 10^{4}\right.$ cells/well cultured in RPMI medium) in a microtiter plates with a flat bottom. Macrophages were then infected with $5 \times 10^{5}$ promastigotes. Plates were incubated at $33^{\circ} \mathrm{C}$ for 1 day and then at $37^{\circ} \mathrm{C}$ for another day. After incubation, the culture medium was removed and washed with buffered RPMI-HEPES ( $\mathrm{pH}$ 7.2) to eliminate any non-internalised 
parasites, after which tempered media RPMI $(100 \mu \mathrm{L})$ was added to each well along with another $100 \mu \mathrm{L}$ of media containing different concentration of drugs or formulations were added. Stock solutions for Miltefosine and BPQ (20 mg mL $\left.{ }^{-1}\right)$ were prepared initially in DMSO and then diluted to $40 \mu \mathrm{g} \mathrm{mL}^{-1}$ with RPMI-HEPES media (pH 6.7), while blank or BPQ SNEDDS and blank or BPQ solid SNEDDS were diluted with RPMI-HEPES media ( $\mathrm{pH} 6.7$ ) at a final concentration of $40 \mu \mathrm{g} \mathrm{mL} \mathrm{m}^{-1}$. Further dilutions of the formulations using the same media were performed and were added up to a final volume of $200 \mu \mathrm{L}$ resulting in a final concentration of $40,20,10,1,0.1$, $0.01,0.001,0.0001,0.00001 \mu \mathrm{g} \mathrm{mL}-1$ per well. The final solvent (DMSO) concentrations never exceeded $0.5 \%(\mathrm{v} / \mathrm{v})$ warranting no effect on parasites proliferation or morphology ${ }^{41}$. Sixteen wells were used as positive untreated control and eight wells as negative uninfected control were adjusted also to a final volume of $200 \mu \mathrm{L}$ with fresh RPMI media and incubated at $37^{\circ} \mathrm{C}$ for 48 hours. The culture medium at different drug concentrations was removed by centrifugation at 3,500 rpm for 5 minutes (Eppendorf 5403 Centrifuge, Eppendorf Iberica, Madrid, Spain) and replaced with lysis solution $(0.02 \%$ sodium dodecyl sulphate in RPMI-HEPES buffer). After 20 minutes, the lysis solution was removed by centrifugation $(3,500 \mathrm{rpm}, 5$ minutes, $\left.4^{\circ} \mathrm{C}\right)$. The supernatants were replaced by fresh Schneider's culture media $(200 \mu \mathrm{L})$. The plates were then incubated at $26^{\circ} \mathrm{C}$ for another 3 days to allow the transformation of viable amastigotes into promastigotes and their proliferation. Finally, resazurin salt $(2.5 \mathrm{mM}, 20 \mu \mathrm{L} /$ well $)$ were added followed by a 3 hour incubation at room temperature and relative fluorescence units were measured as described below. All in vitro experiments were carried out for three times and by triplicate. The $\mathrm{IC}_{50}$ was calculated by Probit analysis and the statistic parameters were computed using SPSS Statistics v17.0 Software. 


\section{Cytotoxicity studies on murine macrophages}

J774 murine macrophages were grown in RPMl 1640 medium supplemented with 10\% heat-inactivated FBS $\left(30 \mathrm{~min}\right.$ at $\left.56^{\circ} \mathrm{C}\right)$, penicillin $\mathrm{G}\left(100 \mathrm{U} \mathrm{mL}^{-1}\right)$ and streptomycin $\left(100 \mu \mathrm{gL}^{-1}\right)$ as previously described by our group ${ }^{40,53}$. Cells in the pre-confluence phase were harvested with $0.03 \%$ EDTA- $0.05 \%$ Trypsin in PBS for 20 minutes and maintained at $37{ }^{\circ} \mathrm{C}$ in a humidified environment with $5 \% \mathrm{CO}_{2}$ (Hucoa-Erlöss, Forma Scientific, Ohio, USA). Cytotoxicity studies were conducted using a resazurin assay as described previously ${ }^{42}$ by determining the reduction of resazurin by measuring the Relative Fluorescence Units (RFU, $535 \mathrm{~nm}$ excitation wavelength and $590 \mathrm{~nm}$ emission wavelength, Infinite 200, Tecan i-Control, Männedorf, Switzerland) as in the promastigotes assay. Growth inhibition (\%) was calculated using Equation 3:

Growth inhibition $(\%)=100-\frac{\mathrm{RFU} \text { treated wells }-\mathrm{RFU} \text { signal to noise }}{\mathrm{RFU} \text { untreated }-\mathrm{RFU} \text { signal to noise }} \times 100$ (Equation 3) Every concentration was assayed three times and controls (medium and drug) were used in each test as blanks and the background was subtracted. Cytotoxicity of the tested drugs/formulations was defined as the $50 \%$ reduction of cell viability of treated culture cells with respect to untreated cells $\left(\mathrm{CC}_{50}\right)$. The selectivity index (SI) was calculated as expressed in Equation 4 42:

$\mathrm{SI}=\frac{C C_{50}}{I C_{50}}$

(Equation 4)

\section{In vivo pharmacokinetic studies of oral BPQ SNEDDS}

All experiments were approved and performed in accordance with the local ethics committee rules (Complutense University of Madrid Institutional Animal Care and Ethics Committee, Madrid, Spain). Male CD-1 outbred mice (6 weeks old) were 
purchased from Harlan Iberica S.A. (Barcelona, Spain) and were housed in groups of 5 in plastic cages in controlled laboratory conditions with ambient temperature and humidity maintained at $\sim 22{ }^{\circ} \mathrm{C}$ and $60 \%$ respectively with a $12 \mathrm{~h}$ light and dark cycle (lights on at 07:00 and off at 19:00). Food and water were available ad libitum and the animals acclimatized for 5-7 days prior to experiments, within the animal house.

CD-1 mice were randomly split into groups $(n=3)$, fasted overnight and dosed with BPQ aqueous suspensions prepared by probe sonication (50\% output, 200 watt for 10 minutes) (11 $\left.\mathrm{mg} \mathrm{mL}^{-1}\right)$ or BPQ SNEDDS $\left(11 \mathrm{mg} \mathrm{mL}^{-1}\right)$ at a BPQ dose of $6 \mathrm{mg} \mathrm{kg}^{-}$ ${ }^{1}$ were administered (as liquid suspensions via oral gavage). Mice were sacrificed at different time points $(1,2,4,7$, and $24 \mathrm{~h})$, and blood was harvested. Plasma was separated by centrifugation (2000 rpm, $15 \mathrm{~min}, 4^{\circ} \mathrm{C}$, Hermle Z323 K centrifuge, VWR, Poole, U.K.), and all tissues were stored at $-50^{\circ} \mathrm{C}$ until analyses could be performed on them.

Plasma samples $(100 \mu \mathrm{L})$ were extracted three times with acetonitrile (100 $\mu \mathrm{L})$. After every extraction the mixture was vortexed (Fisher TopMix Vortex Mixer FB15024, Leicestershire, England) for 5 minutes and the samples were then centrifuged $(8,000$ rpm for 10 minutes) (SciSpin Micro Centrifuge, Shropshire, England). The supernatants were pooled and evaporated to dryness in a concentrator (Savant Integrated SpeedVac System ISS110, Fisher Scientific, Loughborough, UK). Extracted plasma calibration curves were constructed by spiking $(5 \mu \mathrm{L})$ of BPQ stocks in DMSO in CD-1 blank plasma to produce a range of $0.1-50 \mu \mathrm{g} \mathrm{mL}^{-1}$ and were extracted and dried in a similar manner to in vivo plasma samples. Extracted standards and samples were reconstituted with mobile phase $(100 \mu \mathrm{L})$ and were analysed in the HPLC as described above. 


\section{Efficacy in a systemic murine model of Visceral Leishmaniasis}

All experiments were approved and performed in accordance with local ethics committee rules (Complutense University of Madrid Institutional Animal Care and Ethics Committee, Madrid, Spain). BALB/c male mice (20-25 g, 6-7 weeks) were purchased form Harlan Iberica S.A. (Barcelona, Spain) and were housed in groups of 5 in plastic cages under controlled laboratory conditions with ambient temperature and humidity maintained at $\sim 22{ }^{\circ} \mathrm{C}$ and $60 \%$ respectively with a $12 \mathrm{~h}$ light and dark cycle (lights on at 07:00 and off at 19:00). Food and water were available ad libitum and the animals acclimatized for 5-7 days prior to experiments, within the animal house.

The preparation of the parasites and the experimental infection were performed as previously described ${ }^{14}$. Each BALB/c mouse was infected with $10^{7}$ promastigotes by intracardiac route using a syringe with $26 \mathrm{G}$ needle.

Mice were randomly split into groups $(n=8)$. Treatment was started on day 21 postinfection. A negative control (distilled water) and positive control (miltefosine in distilled water $\left.12 \mathrm{mg} \mathrm{kg}^{-1}\right)^{43,44}$ were dosed by oral gavage once daily for 10 consecutive days. Oral BPQ SNEDDS and BPQ Solid SNEDDS (6 mg kg-1) groups were also dosed once daily for 10 consecutive days by oral gavage. Formulations were dosed as liquid suspensions of SNEDDS or solid SNEDDS (powders prior being compressed into tablets) via oral gavage. The final group received a BPQ in DMSO intramuscular dose

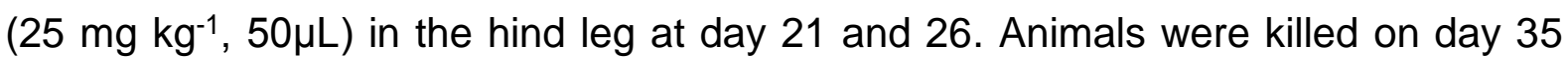
post- infection. Spleen and liver from each animal were aseptically removed and weighted to quantify the parasite burdens. The parasite burden was quantified by the limit dilution assay as previously described ${ }^{14}$ and the percentage suppression of parasite replication (PS) was calculated using the following equation 
$P S=100 \frac{(\mathrm{PC}-\mathrm{PT})}{\mathrm{PC}}$

(Equation 5)

where PC is the number of parasites in the control group per tissue weight $(\mathrm{g})$ and PT is the number of parasites after treatment per tissue weight $(g){ }^{45}$.

\section{Statistical Analysis}

Microsoft Office Excel 2015 was used to analyse all data and graphs were plotted using Microcal Origin 6.0 or GraphPad Prism 7.0. Student t-test or one-way analysis of variance (ANOVA) with Tukey's post-hoc test were performed at a $5 \%$ level of significance using IBM SPSS Statistics 21.

\section{Results}

The optimal microemulsion region was achieved for SNEDDS prepared with BPQ: Labrafil C1944CS: Labrasol: Capryol 90 of 0.01:3:1:5.99 w/w. Drug loading studies with exceeding BPQ amounts (10 mg g ${ }^{-1}, 20 \mathrm{mg} \mathrm{g}^{-1}$ and $\left.50 \mathrm{mg} \mathrm{g}^{-1}\right)$ demonstrated that the maximum loading was $16.7 \pm 2.7 \mathrm{mg} \mathrm{g}^{-1}$, which is comparable to previous published reports of high loading for BPQ (Figure 1A) ${ }^{25}$. Dispersions of BPQ SNEDDS illustrated a quasispherical particle size well below $300 \mathrm{~nm}(206 \pm 15 \mathrm{~nm})$ with low polydispersity $(0.316 \pm 0.014)$ and colloidal stability $(-19.2 \pm 0.6 \mathrm{mV})$ (Figure 1B-E). BPQ content, $\zeta$-potential and particle size remained unaltered over 6 months stored at $40 \pm 2^{\circ} \mathrm{C}$ and $75 \pm 5 \% \mathrm{RH}$ (One Way Anova, $\mathrm{p}<0.05$ ) (Figure SI-2, 1F).

Near complete release $(89.3 \pm 1.9 \%)$ was observed in flow-through cell studies (BP Apparatus IV) with BPQ SNEDDs filled immediate release pullulan capsules within 30 minutes (pH1.2) (Figure 2A). BPQ remained solubilised and available for absorption within all different $\mathrm{pH}$ media tested and complete release was achieved. 
The in vitro dynamic lipid digestion model offers information regarding drug partitioning during hydrolysis of triacylglycerides and quantifying the amount of solubilised drug in the intestinal fluids. Lipolysis was initiated by the addition of the lipase suspension after which the lipolytic process was monitored as a function of time. Calcium chloride solution was added continuously to control the accumulation of fatty acids in the medium by forming insoluble calcium FA soaps, which precipitate thus removing FA from the system and preventing accumulation 33,46 . At 30 minutes, $70 \%$ of $\mathrm{BPQ}$ remained solubilised and un-precipitated, and thus available for absorption, while more than $10 \%(10.4+/-1.25 \%)$ was available for absorption even after 90 minutes, which is almost double previous reports available in literature for orally bioavailable self-emulsifying drug delivery systems (Figure 2B) ${ }^{47}$.

Solid SNEDDS were prepared by adsorption of BPQ loaded SNEDDS on low molecular glycol chitosan polymers that were suspended in water and lyophilised to yield BPQ loaded solid SNEDDS. (BPQ solid SNEDDS). PXRD studies illustrated that BPQ exhibits Bragg peaks characteristic of crystalline material even after lyophilisation (Figure $3 \mathrm{~A}, 3 \mathrm{~B}$ ) that are characterised by a strong endothermic peak at $183^{\circ} \mathrm{C}$ corresponding to the melting point of the drug in DSC studies (Figure 4A-a, 4A-b). BPQ's PXRD spectra is similar to previous reported values ${ }^{48}$ but the difference in Bragg peak intensities is indicative of narrower BPQ plate crystals [Plane $001\left(2 \theta^{\circ}\right.$ :

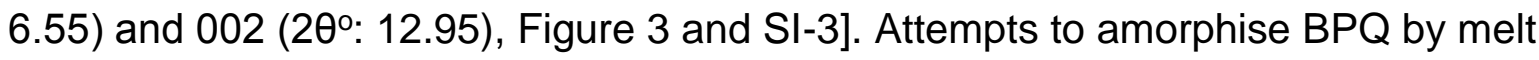


A.

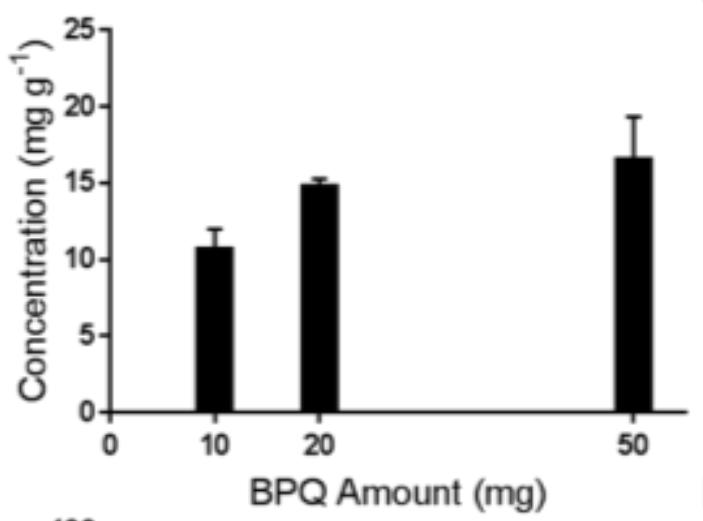

B.

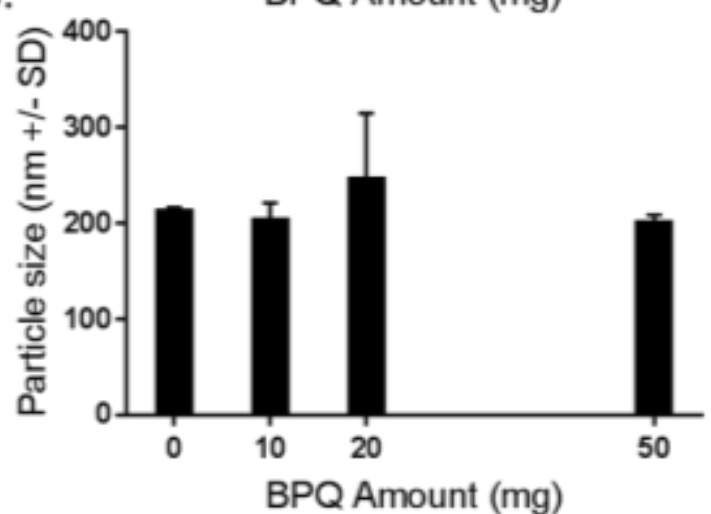

E.

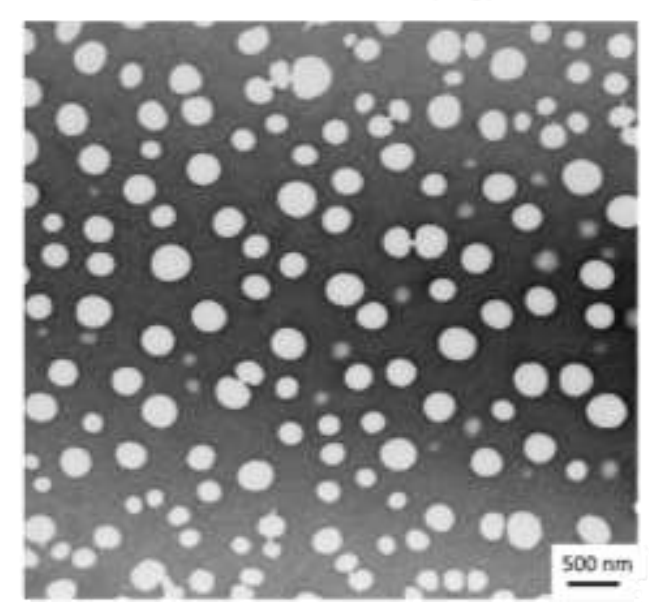

C.

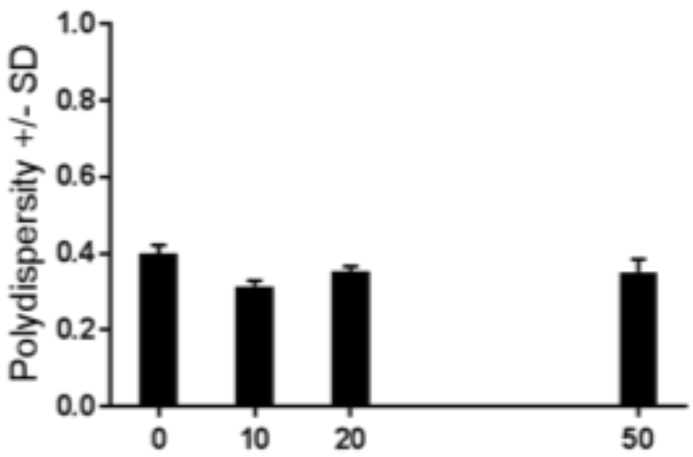

D.

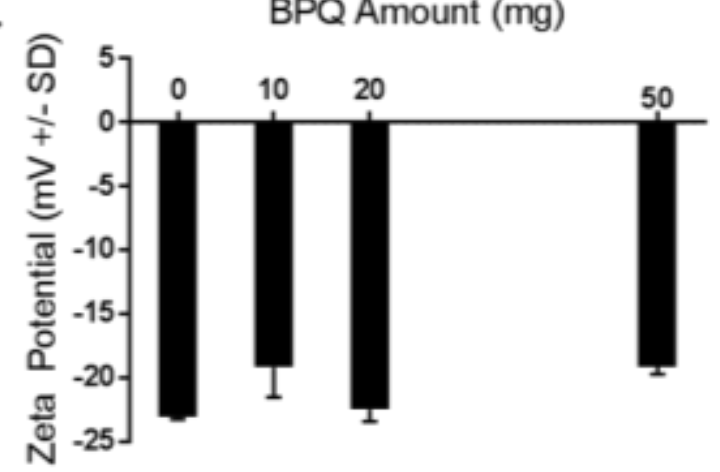

F.

BPQ Amount (mg)

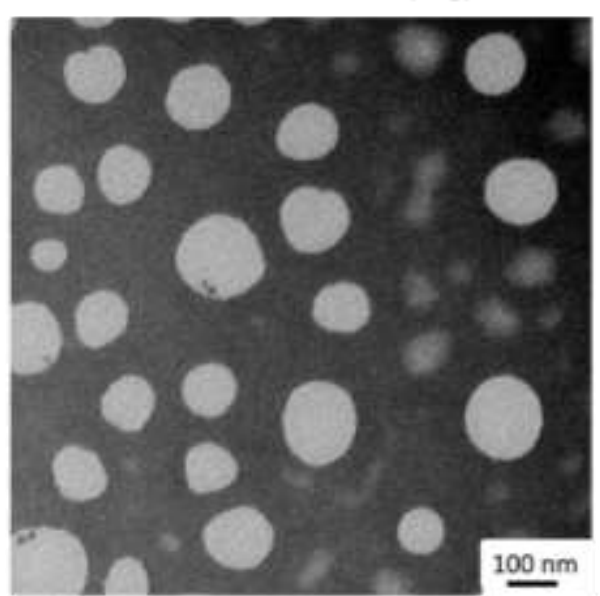

Figure 1. BPQ SNEDDS characteristics; A: Drug Entrapment Studies, B-D: Particle size, polydispersity and zeta-potential of blank and BPQ SNEDDS $(n=3)$ respectively, E-F: TEM of BPQ SNEDDS (1:200 v/v aqueous dilutions) fresh and after 6 weeks storage at $40^{\circ} \mathrm{C}$ and $75 \%$ relative humidity (Bar: 500 and $100 \mathrm{~nm}$ respectively).

quenching technique (heating up to $200^{\circ} \mathrm{C}$ and fast cooling by immersion in liquid nitrogen) was not successful with characteristic BPQ Bragg peaks diminished in intensity but not abolished (Figure 3C). BPQ solid SNEDDS resulted in fewer Bragg peaks which were broader and of lower intensity which are related to other excipients (such as lactose and croscarmellose sodium) (Figure 3D-H). The absence of the 
A.

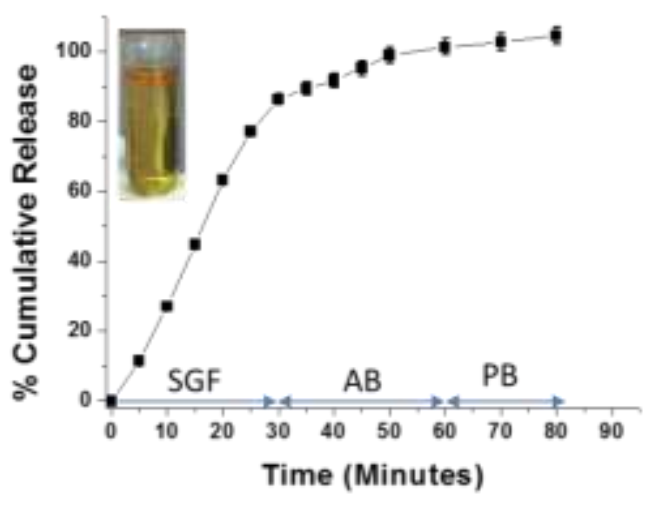

C.

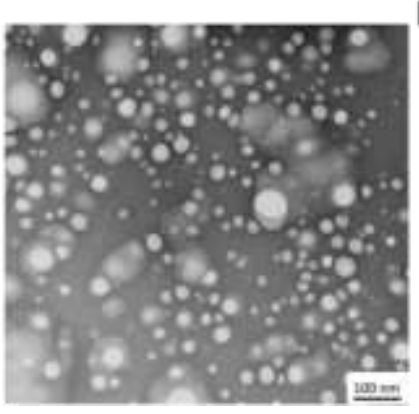

B.

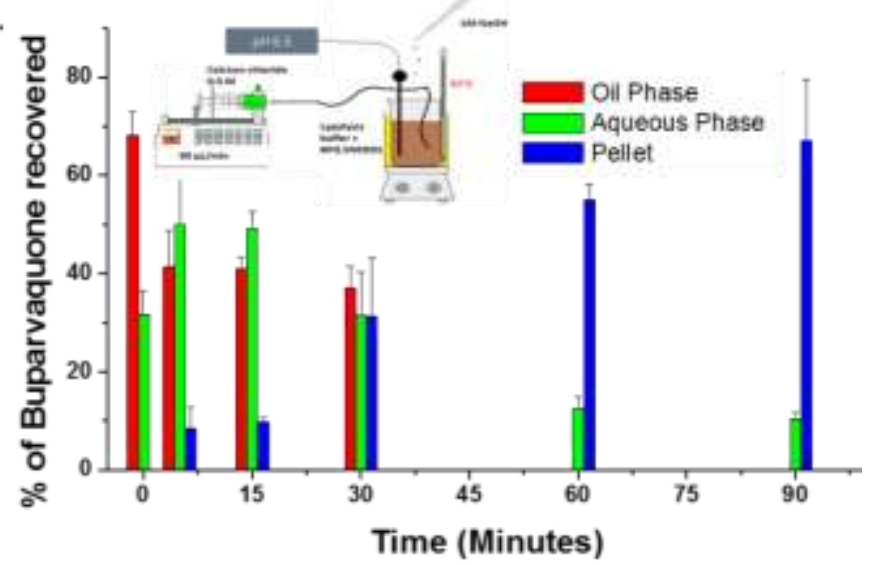

E.
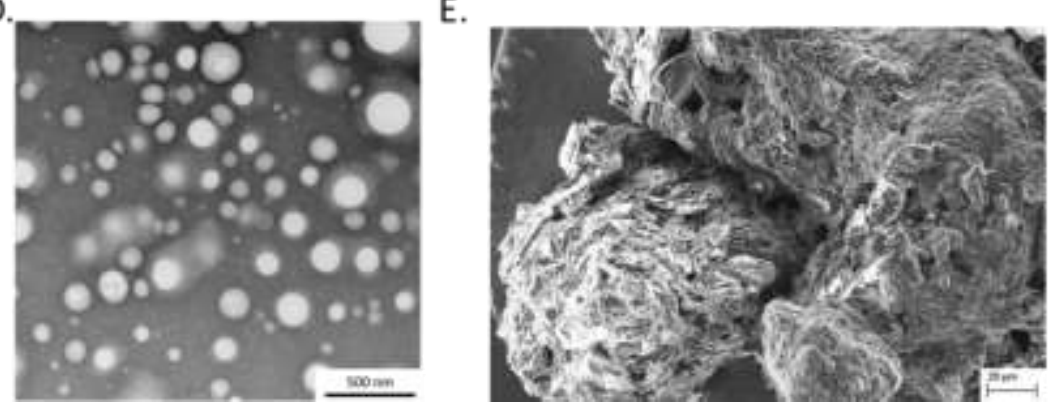

Figure 2. A. Cumulative BPQ release from BPQ SNEDDs pullulan capsules in flowthrough cell studies (Simulated Gastric Fluid (SGF) pH 1.2: 0-30min, Acetate Buffer (AB) $\mathrm{pH}$ 4.5: 30-60 min, Phosphate Buffer (PB) pH 6.8: 60-80 min) ( $\mathrm{n}=3)$, B: In vitro lipolysis of BPQ - SNEDDs with red, green and blue bars illustrating the \% of BPQ recovered in the oil phase, aqueous supernatant and pellet respectively $(n=3)$. C-D: TEM images of BPQ solid SNEDDs aqueous dispersions $\left(1 \mathrm{mg} \mathrm{mL}^{-1}\right.$, Bar: $100 \mathrm{~nm}$ and $500 \mathrm{~nm}$ respectively). E: SEM images of BPQ solid SNEDDS (Magnification: 500x, $5.00 \mathrm{KV}$, Bar $20 \mu \mathrm{m})$.

characteristic peaks of $\mathrm{BPQ}\left(2 \theta^{\circ}: 6.55\right.$ and 12.95$)$ points towards the amorphisation of BPQ (Figure 3H). Additionally, DSC analysis of BPQ solid SNEDDS illustrated no peak at $183^{\circ} \mathrm{C}, \mathrm{BPQ}$ 's characteristic melting endotherm, suggesting also complete amorphisation of BPQ (Figure 4A, Table SI-1). This is further supported by studies of physical mixtures of lyophilised BPQ and blank SNEDDS adsorbed on low molecular weight glycol chitosan $(1: 10 \mathrm{w} / \mathrm{w})$, which indicated a peak at $183^{\circ} \mathrm{C}$ and the characteristic BPQ Bragg peaks of crystalline BPQ (Figure $3 \mathrm{I}, 3 \mathrm{~J}$ and $4 \mathrm{H}$ ). 


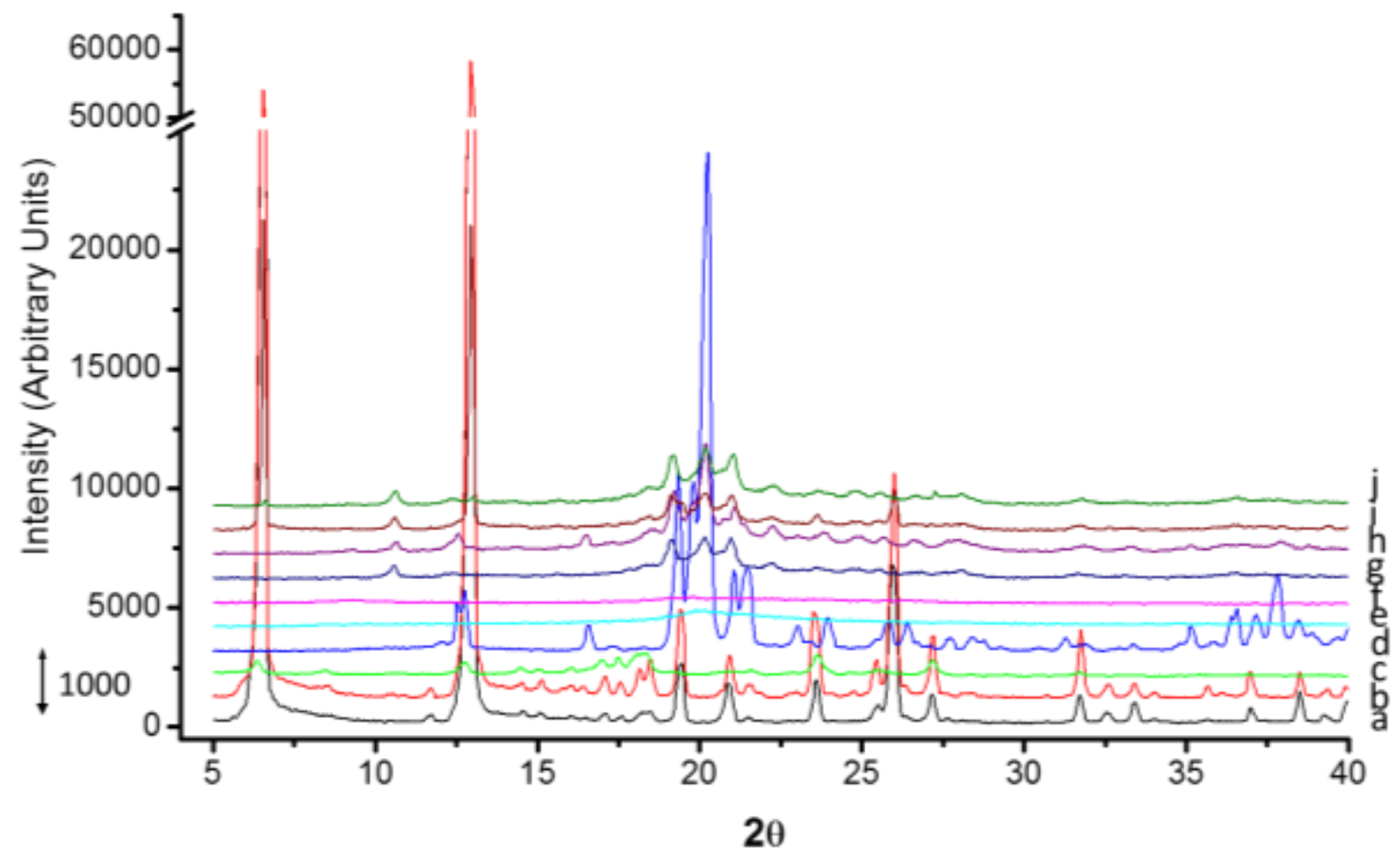

Figure 3. PXRD analysis of prepared blank and BPQ solid SNEDDS along with physical mixtures of BPQ with blank solid SNEDDS; BPQ (a), BPQ lyophilised (b), BPQ amorphised (c), Lactose (d), Croscarmellose Sodium (e), Glycol chitosan (14 $24 \mathrm{kDa}$ ) (f), Blank solid SNEDDS (g), BPQ solid SNEDDS (h), Physical mixture of crystalline BPQ with blank solid SNEDDS (1:10 w/w) (i), Physical mixture of crystalline BPQ with blank solid SNEDDS (1:100 w/w) (j).

FT-IR analysis illustrated that BPQ exhibits two characteristics peaks, a sharp peak at $3300 \mathrm{~cm}^{-1}$ attributed to intramolecular hydrogen bonds between the hydroxyl group of $\mathrm{BPQ}$ and a peak at $1694 \mathrm{~cm}^{-1}$ belonging to the quinine carbonyl group (aromatic group with 2 double bonded oxygens) (Figure 4B). Physical mixtures of BPQ and blank SNEDDS adsorbed on low molecular weight glycol chitosan shows a peak at $1639 \mathrm{~cm}^{-}$ 1 indicative of crystalline BPQ. However, the absence of these peaks for BPQ solid SNEDDS points towards the formation of a solid dispersion (Figure 4B), due to hydrogen bonding between hydroxyl groups of glycol chitosan with the quinine carbonyl groups of BPQ and can further explain the enhanced oral bioavailability of $\mathrm{BPQ}{ }^{26,27 .}$ 
A.

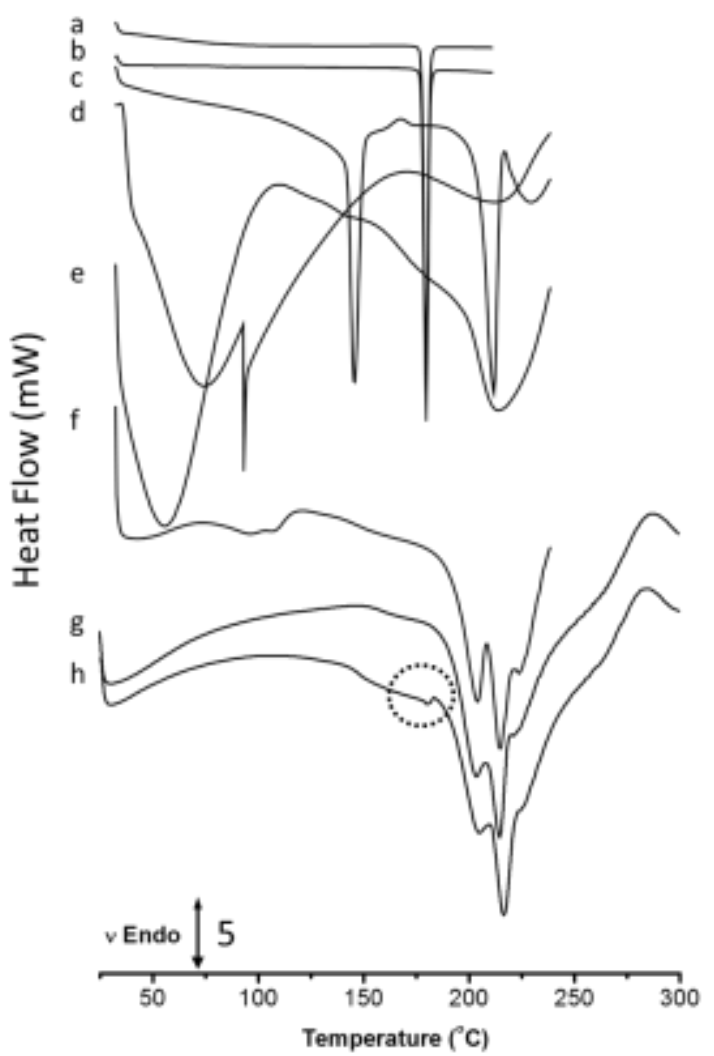

B.

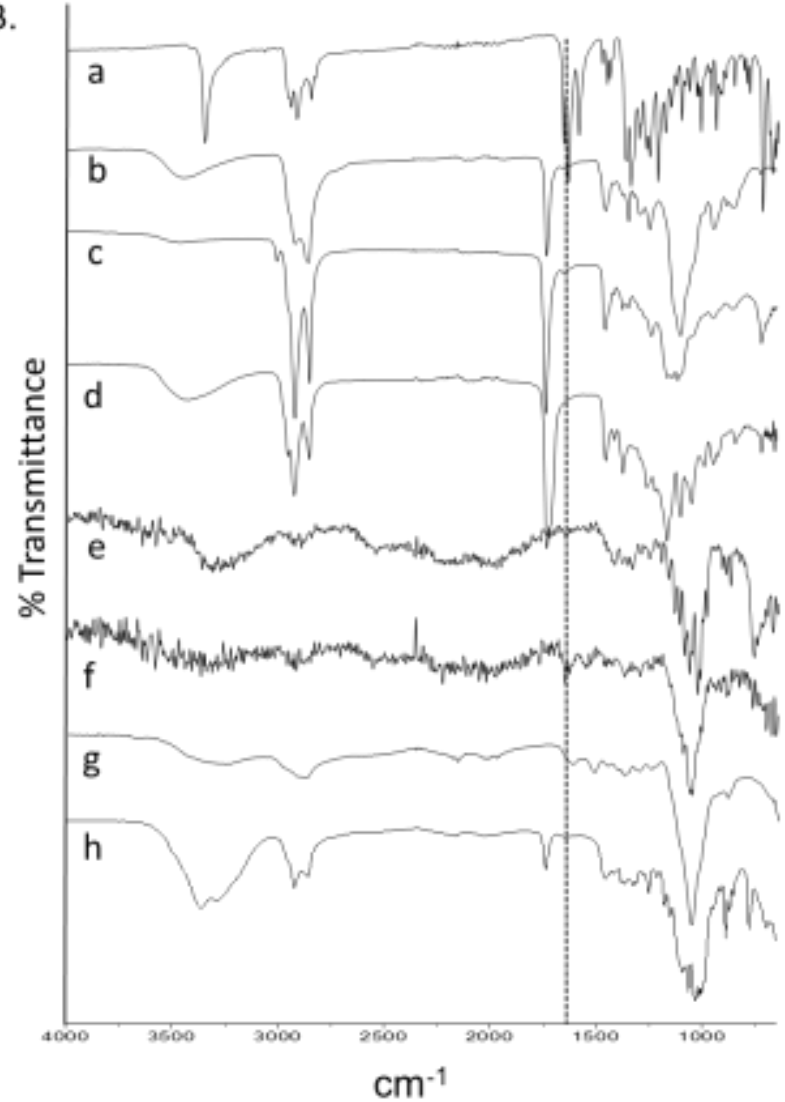

Figure 4. A;DSC thermographs of BPQ (a), BPQ lyophilised (b), Lactose (c), Croscarmellose sodium (d), Glycol chitosan (GC 14-24kDa) (e), BPQ solid SNEDDs $(\mathrm{f})$, Blank solid SNEDDS (g), Physical mixtrure of BPQ $(10 \% \mathrm{w} / \mathrm{w})$ and blank solid SNEDDS (h). B; FT-IR spectra of BPQ (a), Labrasol (b), Labrafil 1944 CS (c), Capryol 90 (d), Lactose (e), Glycol chitosan (GC 14-24 kDa) (f), BPQ solid SNEDDS (GC 14$24 \mathrm{kDa})(\mathrm{g})$, Physical mixture of BPQ $10 \% \mathrm{w} / \mathrm{w}$ and blank solid SNEDDS (GC14) (h). Vertical dotted line is drawn at $1639 \mathrm{~cm}^{-1}$.

Adsorption of SNEDDs onto low molecular weight glycol chitosan (GC) polymers resulted in the formation of porous solids. (Figure 2E). Glycol chitosan is a biocompatible carbohydrate polymer with good solubility in both acidic and neutral $\mathrm{pH}$. Apart from being known to act as a mucoadhesive and oral bioavailability enhancer ${ }^{35}$, 49, 50, chitosans can be used as a disintegrant through swelling and formation of a hydrogel with increasing water content and decreasing hydrogel content from the core to the surface ${ }^{51}$. Formation of a porous matrix aids disintegration and can speed up the dissolution process. Upon reconstitution of the pre-tabletting lyophilised BPQ 
SNEDDS adsorbed on glycol chitosan mix (BPQ solid SNEDDS), a nanosuspension was formed with a particle size similar to the original BPQ SNEDDS in terms of size, polydispersity and zeta potential $(284 \pm 4.6 \mathrm{~nm}, 0.372 \pm 0.006,-28.5 \pm 0.611 \mathrm{mV})$. Thus, release of SNEDDS is possible after adsorption on solid carriers (Figure 2C, 2D).

BPQ SNEDDS adsorbed on low molecular weight glycol chitosan and croscarmellose sodium resulted in a pre-tabletting mix with passable flow characteristics (Hausner ratio and the compressibility index was calculated to be $1.34 \pm 0.03$ and $25.2 \pm 1.52 \%$ respectively ${ }^{52}$. Friability tests of BPQ solid SNEDDS tablets demonstrated losses of $0.89 \pm 0.03 \%$, which are not greater than 1.0 per cent and are acceptable according to BP standards. BPQ solid SNEDDS tablets illustrated a uniformity of content of 87.6 $\pm 3.6 \%$ which is on the lower end of BP limits (85-115\%) with none of the six tested tablets being below $75 \%$. Compressed tablets ( $275 \pm 10.79 \mathrm{mg}, 1 \mathrm{bar}, 10 \mathrm{sec}$ ) were able to disintegrate completely in less than 8 minutes. Premixing the croscarmellose sodium with the glycol chitosan prior to adsorbing the drug loaded SNEDDS is critical in passing pharmacopoeia disintegration tests as simply adding it in the end resulted in times longer than 15 minutes. Additionally, the molecular weight of the carrier polymer (glycol chitosan) is important; using high molecular weight glycol chitosan polymers $(\sim 150 \mathrm{kDa})$ resulted in disintegration times well above 15 minutes irrespectively of the inclusion of a superdisintegrant and in the formation of viscous gels on the surface of the tablets that did not allow water penetration within the core of the tablet and the release of the SNEDDS from the solid carrier posing as a diffusion barrier (data not shown). Dissolution studies illustrated that $47.6 \pm 14.7 \% \mathrm{BPQ}$ was released within 60 minutes in simulated gastric fluids in the absence of pepsin (SGF) and complete release was achieved within 30 minutes after the $\mathrm{pH}$ was raised to 6.8 
(Figure 5). Thus, the complete release of BPQ from solid dosage forms of BPQ nanomedicines and avoiding its precipitation in the absorptive part of the gastrointestinal tract are likely indicators of enhance oral bioavailability of BCS Class Il drugs such as BPQ.

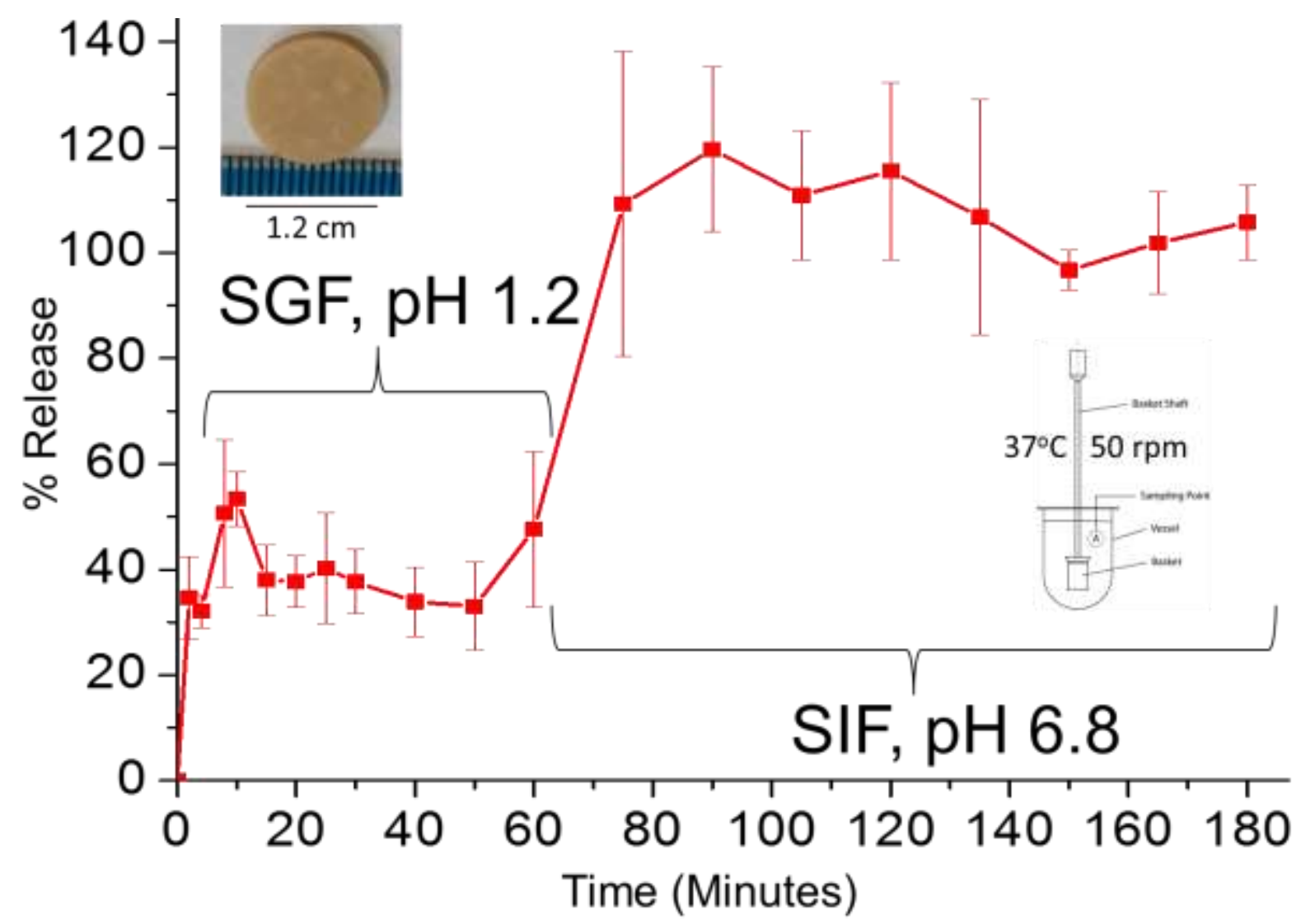

Figure 5. Dissolution of BPQ solid SNEDDS tablets in simulated gastrointestinal tract fluids (SGF: simulated gastric fluid with no pepsin, and SIF: simulated intestinal fluid with no pancreatin).

BPQ SNEDDS and BPQ solid SNEDDS showed potent in vitro efficacy in the nanomolar range against $L$ Infantum amastigotes similar to BPQ in DMSO $\left(\mathrm{IC}_{50}\right.$ for all $<37 \mathrm{nM}$ ), while having negligible cytoxocity against murine macrophages (Table 1). The activity of the blank SNEDDS can be attributed to inclusion of Labrasol. We have recently reported that Labrasol (a mixed ester of capric and caprylic acid) possesses high antileishmanial activity in the micromolar range with acceptable cytotoxicity against murine macrophages ${ }^{53}$. Labrafil on the other hand did not illustrate activity 
against Leishmania parasites similarly to low molecular weight glycol chitosan polymers ${ }^{53}$. Both BPQ and BPQ SNEDDS illustrated a high selectivity index that are well above DNDi (Drug for Neglected Diseases initiative) accepted hit selection levels $(<10 \mu \mathrm{M}, \mathrm{Sl}>10)^{54}$.

Table 1. In vitro activity in L. Infantum amastigotes

\begin{tabular}{|c|c|c|c|c|c|}
\hline Drug/Formulation & $\begin{array}{c}\text { L. Infantum } \\
\text { Promastigotes } \\
\left(\mathrm{IC}_{50} \mathrm{\mu g} \mathrm{mL}^{-1}\right)\end{array}$ & $S I$ & $\begin{array}{l}\text { L. Infantum } \\
\text { Amastigotes } \\
\left(\mathrm{IC}_{50} \mathrm{\mu g} \mathrm{mL}^{-1}\right)\end{array}$ & $S I$ & $\begin{array}{c}J 774 \\
\mathrm{CC}_{50}(\mu \mathrm{g} \\
\left.\mathrm{mL}^{-1}\right)\end{array}$ \\
\hline $\begin{array}{l}\text { Buparvaquone (in } \\
\text { DMSO) }\end{array}$ & $<0.012$ & $>2000$ & $<0.005$ & 5000 & $>25$ \\
\hline $\begin{array}{l}\text { Miltefosine (in } \\
\text { DMSO) }\end{array}$ & $7.19 \pm 0.6$ & 7.7 & $23.7 \pm 1.78$ & 2.3 & $\begin{array}{c}55.4 \pm \\
4.19\end{array}$ \\
\hline Blank SNEDDS & $1.26 \pm 0.10$ & 14.2 & $9.5 \pm 1.9$ & 1.9 & $\begin{array}{r}18.18 \\
\pm 1.04\end{array}$ \\
\hline BPQ SNEDDS & $3.3 \pm 0.09$ & 7.6 & $0.093 \pm 0.053$ & 268.8 & $>25$ \\
\hline $\begin{array}{l}\text { BPQ Solid } \\
\text { SNEDDS }\end{array}$ & $<0.012$ & NS & $<0.005$ & NS & NS \\
\hline
\end{tabular}

Based on this encouraging in vitro results, we decided to conduct an in vivo pharmacokinetic study in healthy mice and efficacy studies in L. infantum infected mice. There are only a few published reports regarding the efficacy or the pharmacokinetic studies of BPQ administered orally. Early reports conducted in a $\mathrm{BALB} / \mathrm{c}$ mouse model illustrated that oral treatment of $\mathrm{BPQ}$ formulated in $0.25 \%$ Cellacol (Methylcellulose) at a dose of $100 \mathrm{mg} / \mathrm{kg} /$ day over five days resulted in $43 \%$ 
suppression of liver amastigotes ${ }^{24}$, while treatment of infected beagles with $(5 \mathrm{mg} \mathrm{kg}$ ${ }^{1}$ day $^{-1}$ for 5 days intramuscularly (IM)) failed to halt the progression of the disease ${ }^{55}$. Pharmacokinetic parameters after oral administration have only been published in a recent study conducted in rabbits that received orally a dose of $3.0 \mathrm{mg} \mathrm{kg}^{-1}$ of BPQ entrapped in SMEDDS prepared from Capryol 90: Cremophor El: Labrasol 9.82:70.72:17.68 w/w) (Cmax: $102 \mathrm{ng} \mathrm{ml}^{-1}$, Tmax: 3.17 hours, $\mathrm{t}_{1 / 2}: 26.44$ hours, AUC 0-60h: $\left.2.43 \mu \mathrm{g} \mathrm{h} \mathrm{ml}^{-1}\right)^{25}$. We decided to utilise a dose of $6 \mathrm{mg} \mathrm{kg}^{-1}$ for a single dose oral pharmacokinetic studies in mice and to utilise this dose over 10 days for our efficacy studies according to the DNDi Target Product Profile for Visceral Leishmaniasis ${ }^{23}$. BPQ SNEDDS resulted in $A^{-} C_{0-24 h}$ of $10.12 \mu \mathrm{g} \mathrm{ml}^{-1}$ and a $\mathrm{Cmax}_{\text {of }} 0.639 \mathrm{\mu g} \mathrm{mL}^{-1}$ which are at least four fold higher than previous published reports for self-emulsifying systems ${ }^{25}$, while also significantly enhancing the bioavailability of BPQ compared to probe sonicated aqueous dispersions of BPQ after oral administration (55\% increase in plasma $\mathrm{AUC}_{0-24)}$ (Figure 6). The latter is difficult to compare as the oral bioavaibility of BPQ alone has not been previously published. Additionally, BPQ SNEDDS illustrated an enhanced half-life compared to BPQ probe sonicated aqueous suspensions (46.374 versus 14.415 hours). In a systemic murine model of visceral leishmaniasis, BPQ SNEDDS and BPQ solid SNEDDS (orally, $6 \mathrm{mg} \mathrm{kg}^{-1}$ over 10 daily doses) and BPQ in DMSO (IM, $25 \mathrm{mg} \mathrm{kg}^{-1}$, one dose on day 1 of treatment and one dose at day 5 of treatment) showed comparable efficacy to miltefosine (orally, $12 \mathrm{mg}$ $\mathrm{kg}^{-1}$ over 10 daily doses) in inhibiting parasite replication in the spleen at a dose that is shown to be effective in mice ${ }^{43}$ (Figure $7, \mathrm{SI}-7$ ). However, BPQ formulations were more variable in inhibiting parasite replication in the liver. 


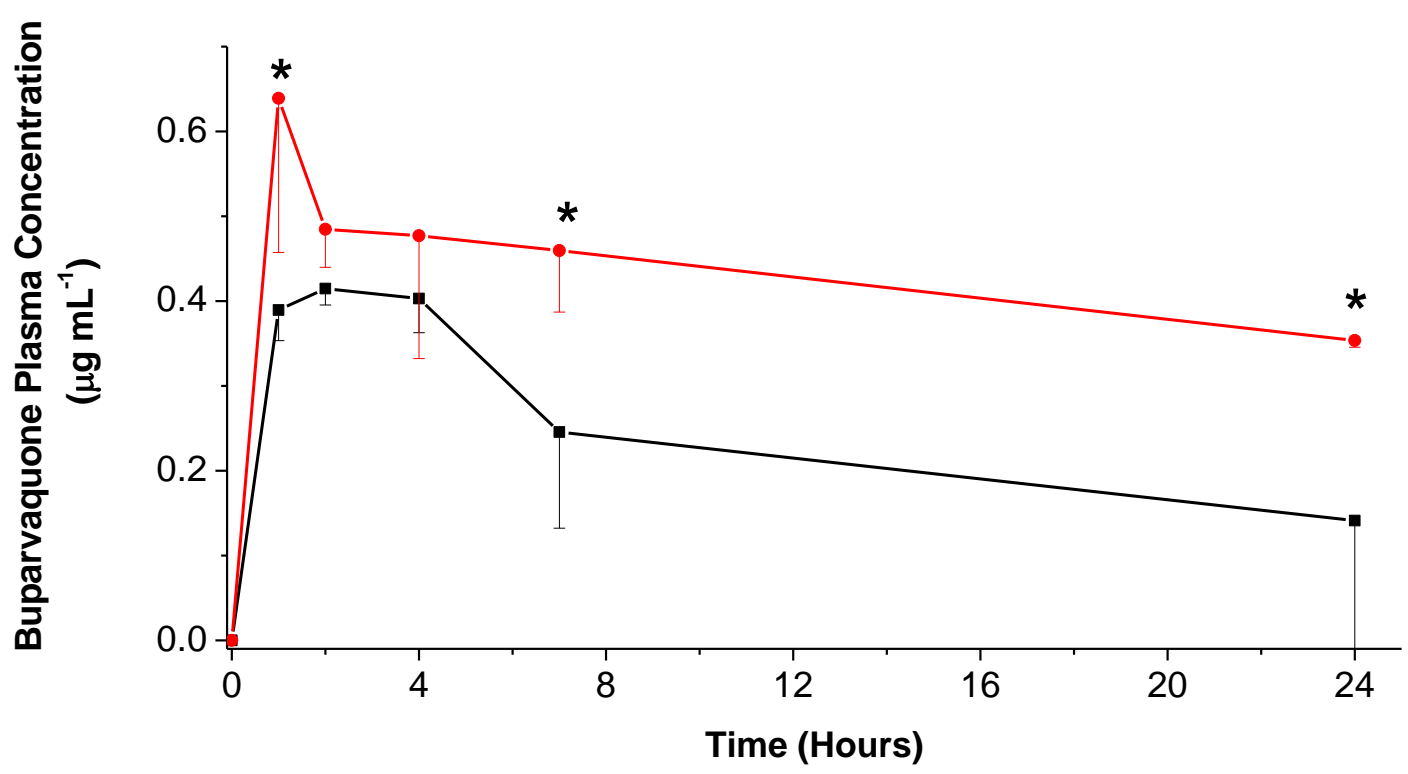

Figure 6. Buparvaquone (BPQ) plasma concentration after oral administration of BPQ (aqueous suspension - probe sonicated, black squares) and BPQ SNEDDS (red circles) formulations $\left(11 \mathrm{mg} \mathrm{g}^{-1}\right)$ to CD-1 mice $(n=3)$. Student t-test: * $p<0.05$; Dose: 6 $\mathrm{mg} \mathrm{kg}^{-1}$ of BPQ.

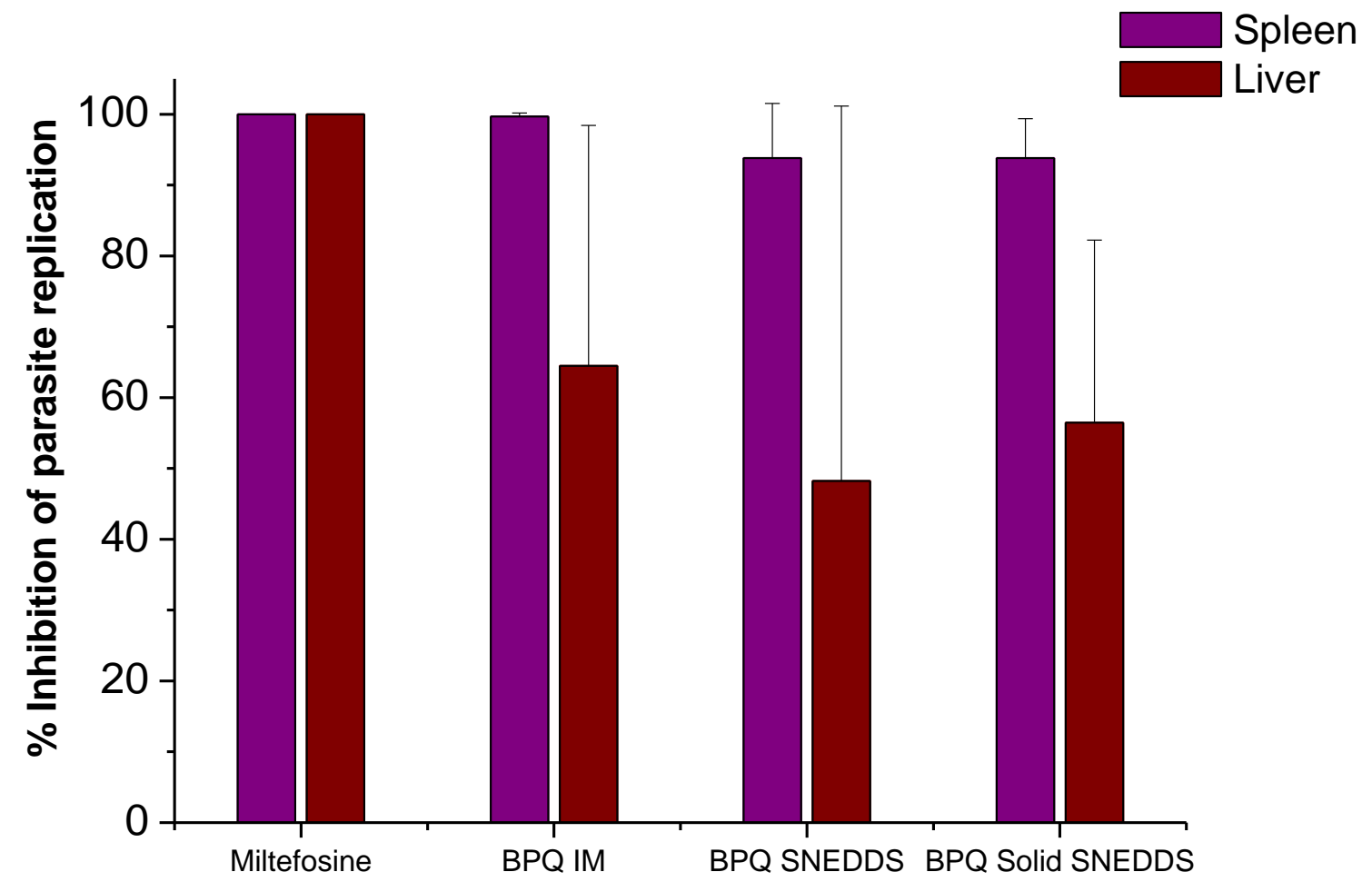

Figure 7. Inhibition of parasite replication after administration of oral miltefosine (12 $\mathrm{mg} \mathrm{kg}^{-1}$ day $^{-1}$, oral, 10 doses), BPQ intramuscularly (25 mg kg-1, IM, 2 doses) and oral BPQ SNEDDS and BPQ solid SNEDDS (6 mg kg-1 day ${ }^{-1}$, oral, 10 doses) to BALB/c mice $(n=6-8)$. Parasite burden in liver and spleen is described in SI-7. 


\section{Discussion}

Napthoquinones and its derivatives such as BPQ are widely distributed in nature and are known for their antibacterial, antifungal, antiviral, antitumour and antiparasitic effects ${ }^{56}$. Napthoquinones interact with biological targets by forming covalent bonds or via their ability to undergo reversible oxidation-reduction reactions by generation of ROS (reactive oxygen species) by the redox cycle under aerobic conditions, by the inhibition of electron transport. The electron transport chain (ETC) entails the sequential transfer of electrons via multiprotein complexes (complex I to IV) present in the inner mitochondrial membrane with electron transfer between several complexes being mediated by ubiquinone ${ }^{57}$. Compounds that are analogs of ubiquinone that can act as competitive inhibitors of cytochrome bc1 by complexing to the Qi ubiquinone binding site (one of two ubiquinone binding sites on cytochrome bc1 and the one that is oriented toward the luminal side of the inner mitochondrial membrane, as distinct from the Qo site, which is oriented toward the external surface of that membrane) ${ }^{57}$. Studies on promastigotes and amastigotes of several species of Leishmania indicated that ETC is critical for the survival of these pathogens as application of antimycin A, an inhibitor of cytochrome bc1, or cyanide, an inhibitor of complex IV, resulted in death of promastigotes or amastigotes ${ }^{57-59}$. BPQ has been recently shown to be a potent inhibitor of cytochrome bc1 activity and the ETC, ATP production and intracellular amastigote growth at nanomolar concentrations ${ }^{24,57}$.

The activity of BPQ against Leishmania donovani was first reported in $1992{ }^{24}$ while BPQ has antiprotozoal activity against a number of parasite including Theileria parva, Leishmania spp., Trypanosoma cruzi, Trypanosoma brucei, Plasmodium falciparum, Eimeria tenella $60 . \mathrm{BPQ}$ is currently used only in veterinary medicine as antitheilerial to treat East Coast fever in cattle in Eastern and Southern Africa (Butalex (C) formulated 
as an IM injection in DMSO $\left(2.5 \mathrm{mg} \mathrm{kg}^{-1}\right)^{60}$. BPQ is characterised by a long plasma half-life of at least 7 days and an oral LD 50 greater than $8 \mathrm{~g} \mathrm{~kg}^{-1}$ in rats ${ }^{60}$ and efficacy against $L$. Donovani amastigotes in the nanomolar range (ED50: $0.005 \mu \mathrm{M}){ }^{24}$. The methylene group linking the cyclohexyl moiety to the quinone nucleus is particularly important for antileishmanial activity as is the presence of a lipophilic subtituent at the 4'-position of the cyclohexyl ring ${ }^{24}$. In vivo BPQ is efficacious against L.donovani in a BALB/c mouse model and following $100 \mathrm{mg} \mathrm{kg}^{-1}$ for 5 days after oral administration resulted in $43.4 \%$ suppression of liver amastigotes (BPQ suspended in $0.25 \%$ cellacol). The disappointing in vivo data, when compared to in vitro potency was attributed to low plasma levels in the experimental animals partly due to the limited solubility of $\mathrm{BPQ}(<30 \mathrm{ng} / \mathrm{mL}$ over a $\mathrm{pH}$ range of $3.0-7.4)$ and high lipophilicity ${ }^{26}$. Permeability studies using in-situ perfused rat intestine classified BPQ as highly permeable with apparent permeability value (Papp) of $0.912 \times 10^{-4} \mathrm{~cm} \mathrm{sec}^{-1} \mathrm{compared}$ to propranolol which is a high permeability marker with Papp value of $0.716 \times 10^{-4} \mathrm{~cm}$ $\sec ^{-1} 61,62$. Thus BPQ permeability is limited mostly by its aqueous solubility and precipitation in the gastrointestinal tracts typical of BCS Class II drugs and strategies to improve its dissolution are likely to have a positive effect in increasing its oral bioavailability.

Although several strategies have been developed to increase BPQ's bioavailbility, only self-emulsifying drug delivery systems (SEDDS) have shown some promise towards orally BPQ formulations ${ }^{25}$. Lipid-based nano-enabled delivery systems are receiving increasingly interest in the delivery of poorly soluble antiparasitic drugs because they possess no or limited biotoxicity, they are able to enhance the solubilisation capacity of the poorly soluble in the gastrointestinal fluids (due to their small size and large surface area to mass ratio) and are able to enhance transcellular 
and lymphatic oral absorption ${ }^{47}$. Liposomal formulations are naturally taken up by macrophages via phagocytosis potentially delivering the antiparasitic drugs to their target site. However, liposomes are usually poorly loaded and are unstable in the gastrointestinal tract. SEDDS are able to overcome these problems by ensuring high drug loading and stability in gastrointestinal media, enhancing drug dissolution and increase the rate and extent of absorption, whilst they are easily scalable and amenable to low cost industrial manufacture. Excipients used in SEDDS are usually GRAS without the need for further toxicology to be converted from a colloidal into a solid dosage form via melt granulation, lyophilisation, spray-drying, physical adsorption onto solid carriers or simply by being filled into capsules ${ }^{63}$. Here we presented a lipid-based formulation of BPQ that possesses good loading, stability at tropical temperatures and simulated gastrointestinal media, ability to release BPQ avoiding precipitation in the aqueous environment of the GIT, enhanced oral bioavailability while being efficacious in reducing parasite load in the spleen similar to miltefosine (only orally VL licensed drug) and near 50\% inhibition of liver parasite load in murine models after 10 days or oral administration. Adsorption of this lipid based formulation on solid carriers and lyophilisation resulted in a solid-dosage form that can be readily translatable with immediate release and enhanced dissolution in the GIT. BPQ SNEDDS were isotropic mixtures of Labrasol: Capryol 90 and Labrafil 1944CS that yield a stable nanoemulsions upon dilution in aqueous media with a particle size well below $300 \mathrm{~nm}$ of quasispherical shape and good colloidal stability (Figure 1). These excipients were selected based on their HLB values to ensure the production of a clear microemulsion type II but also the solubility of BPQ in them (Labrasol: $17 \pm$ 0.28, Labrafil: $12.39 \pm 0.35$, Capryol $90: 15.58 \pm 0.57$ at $\left.37^{\circ} \mathrm{C}\right)^{25}$. Additionally, we have recently shown that Labrasol, a mixed ester of capric and caprylic acid, was shown to 
possess high antileishmanial activity in the nanomolar range ${ }^{53}$. Inclusion of non-ionic surfactants such as Labrafil 1944CS has also been shown to enable their diffusion through mucous layers ${ }^{64}$.

The near complete release of BPQ from BPQ SNEDDS loaded in pullulan capsules in flow-through cell studies (USP Apparatus IV) supports that BPQ remains solubilised and available for absorption within all different $\mathrm{pH}$ media tested (Figure 2A). The in vitro lipolysis model was used to simulate the in vivo digestion process of lipid-based formulations of BPQ. The $\mathrm{pH}$ was maintained at 6.5 to simulate the fasted conditions of the intestine ${ }^{65}$ and to ensure the activity of pancreatic lipase ${ }^{66}$. Lipid digestion was started by the addition of the lipase enzyme while the liberation of free FA that causes a drop in $\mathrm{pH}$ was counteracted by addition of sodium hydroxide. As a dynamic lipolysis model was used, the continuous addition of calcium chloride solution controlled the accumulation of FA on the surface of the emulsions particles by forming insoluble calcium FA soaps, which precipitate and are removed from the system ${ }^{33}$. Although lipid glycerides with medium chain fatty acids are subjected to faster lipolysis ${ }^{47}, 70 \%$ of $\mathrm{BPQ}$ remained solubilised avoiding precipitation in the Gl tract available for absorption after 30 minutes and more than $10 \%$ was available for absorption even after 90 minutes which is double previous published reports for orally bioavailable SEDDS (Figure 2B) ${ }^{47}$. This is important to note, as our nanoemulsions were well below $300 \mathrm{~nm}$ and it is known that reducing the size of the emulsion increases the specific surface area of the lipids and thus the action of pancreatic lipases ${ }^{67}$. The presence of $\mathrm{BPQ}$ as an oil layer for 30 minutes can allow BPQ to be absorbed at a later stage enhancing the gastric residence time ${ }^{68}$.

The Target Product (treatment) Profile (TPP) for VL is based around the development of a safe, effective, oral, short-course (11 days maximum) VL drug formulations to 
replace current treatments. Ideally a solid dosage form with enhanced stability in tropical temperatures is likely to be more cost-effective. BPQ-SNEDDS illustrated stability up to 6 months in tropical conditions $\left(40^{\circ} \mathrm{C}, 75 \%\right.$ relative humidity) in terms of drug content, particle size, colloidal stability and morphology (Figure SI-2). In an attempt to reduce the cost of a liquid filled capsule, we adsorbed BPQ SNEDDS on solid carriers to produce conventional solid dosage forms such as tablets. Aqueous dispersions of BPQ solid SNEDDS resulted in nanoemulsions of similar size and morphology (Figure 2C, 2D). Although the flow properties of BPQ solid SNEDDS could be improved by the addition of a glidant or use of micronized lactose, tablets were produced that passed pharmacopoeia disintegration and dissolution tests for immediate release tablets. Dissolution studies illustrated a $\mathrm{pH}$ mechanism of release with an initial burst release of BPQ from tablets in SGF that remained constant over 60 minutes that was followed by a complete release of the drug when the $\mathrm{pH}$ was raised to 6.8 (Figure 6). Low molecular weight glycol chitosan polymers are biocompatible carbohydrate polymers with good solubility in both acidic and neutral $\mathrm{pH}^{69}, 70$. Chitosans are known to swell when exposed to aqueous media and the inclusion of a super-disintegrant enables the formation of pores to allow water penetration within the polymer gel structure. However, superdisintegrants are known to behave differently in acidic and neutral media ${ }^{71}$. Croscarmellose sodium (Ac-Di-Sol (C) has been shown to promote faster dissolution in neutral media than acidic ${ }^{72}$ which can explain dissolution behaviour observed and favouring of BPQ release in simulated intestinal media.

Solid state characterisation of BPQ solid SNEDDS is critical to understand the interaction of BPQ with the solid carriers at the molecular level and assess whether the formation of a $\mathrm{BPQ}$ solid dispersion or $\mathrm{BPQ}$ amorphisation was possible. 
Lyophilisation of BPQ in a method used for the production of solid SNEDDS did not result in amorphous BPQ (Figure 3b, 4b). Similarly, attempts to amorphise BPQ by melt quenching which has been previously reported as a method to amorphise BCS Class II drugs were unsuccessful (Figure 3c). This can be explained as PXRD analysis indicated a prominent Bragg peak at $6.552 \theta^{\circ}$ which correlates with a hydrophobic surface formed by aromatic rings of BPQ (Plane 001) (Figure SI-3). Additionally, the intensity of the Bragg peak at $12.9520^{\circ}$ corresponding to plane 002 (location of carbonyl groups) was also high, which indicates that $\mathrm{h}$-bonding is likely to occur between the drug and surrounding hydrogen group donors such as glycol chitosan. PXRD, DSC and FT-IR studies indicate the formation of an amorphous BPQ solid dispersion (a product formed by the conversion of a fluid drug-carrier combination to the solid state ${ }^{73}$ ) which is important towards improved oral bioavailability. FT-IR analysis also pointed to the formation of hydrogen bonding between the hydroxyl groups of glycol chitosan polymer chains and the quinine carbonyl group of BPQ as indicated by the abolishment of the $1639 \mathrm{~cm}^{-1}$ peak within the BPQ solid SNEDDS powders.

Entrapment of BPQ within SNEDDS or solid SNEDDS did not affect its in vitro efficacy as BPQ SNEDDS and BPQ solid SNEDDS showed potent in vitro efficacy in the nanomolar range against $L$ Infantum amastigotes similar to BPQ in DMSO (IC 50 for all $<37 \mathrm{nM}$ ) (Table 1). Both BPQ and BPQ SNEDDS showed excellent selectivity for parasite versus mammalian cells with a high selectivity index that are well above DND $i$ (Drug for Neglected Diseases initiative) accepted hit selection levels ${ }^{54}$ and that of miltefosine. The high selectivity of $B P Q$ is expected as there is a high degree of sequence divergence between the Leishmania and human cytochrome b proteins ${ }^{57}$. 
In vivo pharmacokinetic studies demonstrated an earlier onset of action for BPQ SNEDDS compared to BPQ which supports the immediate release and enhanced solubilisation of $\mathrm{BPQ}$ in gastrointestinal media resulting in $\mathrm{AUC}_{0-24}$ that are four fold previous reported values for BPQ SNEDDS after a single oral administration ${ }^{25}$. Although BPQ SNEDDS and BPQ solid SNEDDS were as efficacious as miltefosine in inhibiting parasite replication in the spleen, they were not able to control parasite replication in the liver. Interestingly, oral BPQ SNEDDS and BPQ solid SNEDDS (6 $\mathrm{mg} \mathrm{kg}^{-1}, 10$ oral doses over 10 days) were as efficacious as intramuscular doses of $\mathrm{BPQ}\left(25 \mathrm{mg} \mathrm{kg}^{-1}\right.$, one dose at day 1 and one dose at day 5), indicating that the reasons resulting in limited inhibition of parasite growth in the liver was not related to the oral bioavaibility of $\mathrm{BPQ}$ and amount of $\mathrm{BPQ}$ reaching the liver, but possibly due to metabolism of BPQ in the liver. BPQ has been reported to be metabolised by mouse and rat liver microsomes into an inactive red-coloured metabolic product with a halflife of less than 10 minutes being a substrate for CYP2C9 (half-life 6 minutes) and CYPC19 half-life (4 minutes), while it was also metabolised by CYP3A4 (half-life 50 minutes) $28,74,75$. To overcome this issue and ensure orally bioavailable and efficacious BPQ formulations, further studies of combinations of BPQ with fluconazole, a potent CYP2C9, CYPC19 and CYP3A4 inhibitor shown to be efficacious in the treatment of visceral leishmaniasis 76,77 can be prepared and formulated easily with described SNEDDS platform technology into patient compliant solid dosage forms (SNEDDS filled capsules or tablets). Additionally, preparing SNEDDS with a lower amount of long-chain triglycerides (i.e. Labrafil $1944 \mathrm{CS} \sim 30 \% \mathrm{w} / \mathrm{w}$ of final formulation) and a higher short and medium-chain triglycerides (e.g. Capryol 90 and Labrasol) can potentially decrease the accumulation of the formulation in the liver to reduce BPQ metabolism. 


\section{Conclusions}

Nanotechnology-enabled drug delivery systems offer a tool for overcoming the challenges encountered with current drugs, failed leads such as BPQ and antigens by improving their efficacy (and thus duration of treatment), safety, bioavailability, halflife, while also by enabling controlled release over tailored period of times and specific site-targeted delivery of therapeutics. When made with FDA or EMA approved scalable excipients they can result in medicine that can be taken readily to phase III human studies providing therapeutic solutions to neglected tropical diseases. Our proposed nano-enabled platform technology prepared from GRAS excipients is costeffective while it possesses excellent loading, stability up to 6 months in tropical conditions, in vitro efficacy in the nanomolar range maintaining the solubilisation capacity of BPQ in gastrointestinal media and ensuring high oral bioavailbility for BPQ able to inhibit parasite replication in the spleen and in the liver. Proposed BPQ

SNEDDS and solid SNEDDS are cost-effective, patient compliant and readily translated platform technologies towards a nano-enabled safe and efficacious treatment for parasitic and infectious diseases.

\section{Acknowledgements}

This work was supported by a University of Portsmouth, Research and Development Fund and a Sir Halley Stewart Trust grant (127) provided to Dr Aikaterini Lalatsa. Mr. David McCarthy (UCL School of Pharmacy) is thanked for providing transmission electron microscopy expertise.

\section{References:}

1. Sachs, J. Helping the World's Poorests. The Economist 1999. 
2. McDonald, M. A15 Neglected tropical and zoonotic diseases and their impact on women's and children's health; ISBN-13:978-0-309-18634-6; Institute of Medicine (US) Forum on Microbial Threats: Washington (DC), 2011.

3. WHO Why are some tropical diseases called "neglected". http://www.who.int/features/qa/58/en/ (8th November ),

4. Harries, A. D.; Dye, C. Tuberculosis. Annals of tropical medicine and parasitology 2006, 100, (5-6), 415-31.

5. Jain, K.; Jain, N. K. Novel therapeutic strategies for treatment of visceral leishmaniasis. Drug discovery today 2013, 18, (23-24), 1272-81.

6. WHO Leishmaniasis. http://www.who.int/leishmaniasis/en/ (8th November),

7. Hotez, P. J.; Molyneux, D. H.; Fenwick, A.; Kumaresan, J.; Sachs, S. E.; Sachs, J. D.;

Savioli, L. Control of neglected tropical diseases. The New England journal of medicine 2007, $357,(10), 1018-27$.

8. $\quad$ WHO Weekly epidemiological record; Switzerland, 2002; pp 365-372.

9. Alvar, J.; Velez, I. D.; Bern, C.; Herrero, M.; Desjeux, P.; Cano, J.; Jannin, J.; den Boer, M.; Team, W. H. O. L. C. Leishmaniasis worldwide and global estimates of its incidence. PloS one 2012, 7, (5), e35671.

10. Andreani, G.; Lodge, R.; Richard, D.; Tremblay, M. J. Mechanisms of interaction between protozoan parasites and HIV. Current opinion in HIV and AIDS 2012, 7, (3), 276-82.

11. Diro, E.; Lynen, L.; Ritmeijer, K.; Boelaert, M.; Hailu, A.; van Griensven, J. Visceral Leishmaniasis and HIV coinfection in East Africa. PLoS neglected tropical diseases 2014, 8, (6), e2869.

12. Frezard, F.; Demicheli, C.; Ribeiro, R. R. Pentavalent antimonials: new perspectives for old drugs. Molecules 2009, 14, (7), 2317-36.

13. Serrano, D. R.; Lalatsa, A. Oral Amphtericin B: The journey from bench to market. . Journal of Drug Delivery Science and Technology 2017, 42, 75-83.

14. Serrano, D. R.; Lalatsa, A.; Dea-Ayuela, M. A.; Bilbao-Ramos, P. E.; Garrett, N. L.; Moger, J.; Guarro, J.; Capilla, J.; Ballesteros, M. P.; Schatzlein, A. G.; Bolas, F.; Torrado, J. J.; Uchegbu, I. F. Oral particle uptake and organ targeting drives the activity of amphotericin B nanoparticles. Molecular pharmaceutics 2015, 12, (2), 420-31.

15. Gaboriau, F.; Cheron, M.; Petit, C.; Bolard, J. Heat-induced superaggregation of amphotericin B reduces its in vitro toxicity: a new way to improve its therapeutic index. Antimicrobial agents and chemotherapy 1997, 41, (11), 2345-51.

16. Serrano, D. R.; Hernandez, L.; Fleire, L.; Gonzalez-Alvarez, I.; Montoya, A.; Ballesteros, M. P.; Dea-Ayuela, M. A.; Miro, G.; Bolas-Fernandez, F.; Torrado, J. J. Hemolytic and pharmacokinetic studies of liposomal and particulate amphotericin B formulations. International journal of pharmaceutics 2013, 447, (1-2), 38-46.

17. Pham, T. T.; Loiseau, P. M.; Barratt, G. Strategies for the design of orally bioavailable antileishmanial treatments. International journal of pharmaceutics 2013, 454, (1), 539-52.

18. Dorlo, T. P.; van Thiel, P. P.; Huitema, A. D.; Keizer, R. J.; de Vries, H. J.; Beijnen, J. H.; de Vries, P. J. Pharmacokinetics of miltefosine in Old World cutaneous leishmaniasis patients. Antimicrobial agents and chemotherapy 2008, 52, (8), 2855-60.

19. Mishra, J.; Singh, S. Miltefosine resistance in Leishmania donovani involves suppression of oxidative stress-induced programmed cell death. Experimental parasitology 2013, 135, (2), 397-406.

20. Fairlamb, A. H. Chemotherapy of human African trypanosomiasis: current and future prospects. Trends in parasitology 2003, 19, (11), 488-94.

21. Pecoul, B. New drugs for neglected diseases: from pipeline to patients. PLoS medicine 2004, $1,(1)$, e6. 
22. Freitas-Junior, L. H.; Chatelain, E.; Kim, H. A.; Siqueira-Neto, J. L. Visceral leishmaniasis treatment: What do we have, what do we need and how to deliver it? International journal for parasitology. Drugs and drug resistance 2012, 2, 11-9.

23. DNDi Target Product Profile - Visceral Leishmaniasis. https://www.dndi.org/diseasesprojects/leishmaniasis/tpp-vl/ (19th January),

24. Croft, S. L.; Hogg, J.; Gutteridge, W. E.; Hudson, A. T.; Randall, A. W. The activity of hydroxynaphthoquinones against Leishmania donovani. The Journal of antimicrobial chemotherapy 1992, 30, (6), 827-32.

25. Venkatesh, G.; Majid, M. I.; Mansor, S. M.; Nair, N. K.; Croft, S. L.; Navaratnam, V. In vitro and in vivo evaluation of self-microemulsifying drug delivery system of buparvaquone. Drug development and industrial pharmacy 2010, 36, (6), 735-45.

26. Mantyla, A.; Garnier, T.; Rautio, J.; Nevalainen, T.; Vepsalainen, J.; Koskinen, A.; Croft, S. L.; Jarvinen, T. Synthesis, in vitro evaluation, and antileishmanial activity of watersoluble prodrugs of buparvaquone. Journal of medicinal chemistry 2004, 47, (1), 188-95.

27. Mantyla, A.; Rautio, J.; Nevalainen, T.; Keski-Rahkonen, P.; Vepsalainen, J.; Jarvinen, T. Design, synthesis and in vitro evaluation of novel water-soluble prodrugs of buparvaquone. European journal of pharmaceutical sciences : official journal of the European Federation for Pharmaceutical Sciences 2004, 23, (2), 151-8.

28. Mantyla, A.; Rautio, J.; Nevalainen, T.; Vepsalainen, J.; Juvonen, R.; Kendrick, H.; Garnier, T.; Croft, S. L.; Jarvinen, T. Synthesis and antileishmanial activity of novel buparvaquone oxime derivatives. Bioorganic \& medicinal chemistry 2004, 12, (13), 3497-502. 29. Hernandez-Trejo, N.; Kayser, O.; Steckel, H.; Muller, R. H. Characterization of nebulized buparvaquone nanosuspensions--effect of nebulization technology. Journal of drug targeting 2005, 13, (8-9), 499-507.

30. Muller, R. H.; Jacobs, C. Buparvaquone mucoadhesive nanosuspension: preparation, optimisation and long-term stability. International journal of pharmaceutics 2002, 237, (1-2), 151-61.

31. Jacobs, C.; Kayser, O.; Muller, R. H. Production and characterisation of mucoadhesive nanosuspensions for the formulation of bupravaquone. International journal of pharmaceutics 2001, 214, (1-2), 3-7.

32. Venkatesh, G.; Majid, M. I. A.; Ramanathan, S.; Mansor, S. M.; Nair, N. K.; Croft, S. L.; Navaratnam, V. Optimization and validation of RP-HPLC-UV method with solid-phase extraction for determination of buparvaquone in human and rabbit plasma: application to pharmacokinetic study. Biomedical Chromatography 2008, 22, (5), 535-541.

33. Fatouros, D. G.; Deen, G. R.; Arleth, L.; Bergenstahl, B.; Nielsen, F. S.; Pedersen, J. S.; Mullertz, A. Structural development of self nano emulsifying drug delivery systems (SNEDDS) during in vitro lipid digestion monitored by small-angle X-ray scattering. Pharmaceutical research 2007, 24, (10), 1844-53.

34. Lalatsa, A.; Garrett, N. L.; Ferrarelli, T.; Moger, J.; Schatzlein, A. G.; Uchegbu, I. F. Delivery of peptides to the blood and brain after oral uptake of quaternary ammonium palmitoyl glycol chitosan nanoparticles. Molecular pharmaceutics 2012, 9, (6), 1764-74.

35. Lalatsa, A.; Lee, V.; Malkinson, J. P.; Zloh, M.; Schatzlein, A. G.; Uchegbu, I. F. A prodrug nanoparticle approach for the oral delivery of a hydrophilic peptide, leucine(5)enkephalin, to the brain. Molecular pharmaceutics 2012, 9, (6), 1665-80.

36. Rolon, M.; Serrano, D. R.; Lalatsa, A.; de Pablo, E.; Torrado, J. J.; Ballesteros, M. P.; Healy, A. M.; Vega, C.; Coronel, C.; Bolas-Fernandez, F.; Dea-Ayuela, M. A. Engineering Oral and Parenteral Amorphous Amphotericin B Formulations against Experimental Trypanosoma cruzi Infections. Molecular pharmaceutics 2017, 14, (4), 1095-1106.

37. Serrano, D. R.; Persoons, T.; D'Arcy, D. M.; Galiana, C.; Dea-Ayuela, M. A.; Healy, A. M. Modelling and shadowgraph imaging of cocrystal dissolution and assessment of in vitro 
antimicrobial activity for sulfadimidine/4-aminosalicylic acid cocrystals. European journal of pharmaceutical sciences : official journal of the European Federation for Pharmaceutical Sciences 2016, 89, 125-36.

38. British-Pharmacopoeia British Pharmacopoeia 2018 Online, Appendix XII A. Disintegration. Assessed via: https://www.pharmacopoeia.com/bp-2018/appendices/appendix12/appendix-xii-a--disintegration.html?date=2018-04-01\&text=appendix $+\mathrm{Xii}+\mathrm{a} \quad$ on $(11 / 05 / 2018)$

39. Bilbao-Ramos, P.; Galiana-Rosello, C.; Dea-Ayuela, M. A.; Gonzalez-Alvarez, M.; Vega, C.; Rolon, M.; Perez-Serrano, J.; Bolas-Fernandez, F.; Gonzalez-Rosende, M. E. Nuclease activity and ultrastructural effects of new sulfonamides with anti-leishmanial and trypanocidal activities. Parasitology international 2012, 61, (4), 604-13.

40. Dea-Ayuela, M. A.; Castillo, E.; Gonzalez-Alvarez, M.; Vega, C.; Rolon, M.; BolasFernandez, F.; Borras, J.; Gonzalez-Rosende, M. E. In vivo and in vitro anti-leishmanial activities of 4-nitro-N-pyrimidin- and N-pyrazin-2-ylbenzenesulfonamides, and N2-(4nitrophenyl)-N1-propylglycinamide. Bioorganic \& medicinal chemistry 2009, 17, (21), 744956.

41. Bilbao-Ramos, P.; Sifontes-Rodriguez, S.; Dea-Ayuela, M. A.; Bolas-Fernandez, F. A fluorometric method for evaluation of pharmacological activity against intracellular Leishmania amastigotes. Journal of microbiological methods 2012, 89, (1), 8-11.

42. Galiana-Rosello, C.; Bilbao-Ramos, P.; Dea-Ayuela, M. A.; Rolon, M.; Vega, C.; Bolas-Fernandez, F.; Garcia-Espana, E.; Alfonso, J.; Coronel, C.; Gonzalez-Rosende, M. E. In vitro and in vivo antileishmanial and trypanocidal studies of new N-benzene- and Nnaphthalenesulfonamide derivatives. Journal of medicinal chemistry 2013, 56, (22), 8984-98. 43. Coelho, A. C.; Trinconi, C. T.; Costa, C. H.; Uliana, S. R. In Vitro and In Vivo Miltefosine Susceptibility of a Leishmania amazonensis Isolate from a Patient with Diffuse Cutaneous Leishmaniasis: Follow-Up. PLoS neglected tropical diseases 2016, 10, (7), e0004720.

44. Sanderson, L.; Yardley, V.; Croft, S. L. Activity of anti-cancer protein kinase inhibitors against Leishmania spp. The Journal of antimicrobial chemotherapy 2014, 69, (7), 1888-91.

45. Manandhar, K. D.; Yadav, T. P.; Prajapati, V. K.; Kumar, S.; Rai, M.; Dube, A.; Srivastava, O. N.; Sundar, S. Antileishmanial activity of nano-amphotericin B deoxycholate. The Journal of antimicrobial chemotherapy 2008, 62, (2), 376-80.

46. MacGrecgor, K. J.; Embleton, J. K.; Lacy, A.; Perry, E.; Solomon, L. J.; Seager, H.; Pouton, C. W. Influence of lipolysis on drug absorption from the gastrointestinal tract. . Advanced Drug Delivery Reviews 1997, 25, 33-46.

47. Ibrahim, F.; Gershkovich, P.; Sivak, O.; Wasan, E. K.; Wasan, K. M. Assessment of novel oral lipid-based formulations of amphotericin B using an in vitro lipolysis model. European journal of pharmaceutical sciences : official journal of the European Federation for Pharmaceutical Sciences 2012, 46, (5), 323-8.

48. Gokhale, N. H.; Padhye, S. B.; Croft, S. L.; Kendrick, H. D.; Davies, W.; Anson, C. E.; Powell, A. K. Transition metal complexes of buparvaquone as potent new antimalarial agents. 1. Synthesis, X-ray crystal-structures, electrochemistry and antimalarial activity against Plasmodium falciparum. Journal of inorganic biochemistry 2003, 95, (4), 249-58.

49. Siew, A.; Le, H.; Thiovolet, M.; Gellert, P.; Schatzlein, A.; Uchegbu, I. Enhanced oral absorption of hydrophobic and hydrophilic drugs using quaternary ammonium palmitoyl glycol chitosan nanoparticles. Molecular pharmaceutics 2012, 9, (1), 14-28.

50. Makhlof, A.; Werle, M.; Tozuka, Y.; Takeuchi, H. Nanoparticles of glycol chitosan and its thiolated derivative significantly improved the pulmonary delivery of calcitonin. International journal of pharmaceutics 2010, 397, (1-2), 92-5. 
51. Omidian, H.; Park, K. Swelling agents and devices in oral drug delivery. . Journal of Drug Delivery Science and Technology 2008, 18, (2), 83-93.

52. Shah, R. B.; Tawakkul, M. A.; Khan, M. A. Comparative evaluation of flow for pharmaceutical powders and granules. AAPS PharmSciTech 2008, 9, (1), 250-8.

53. Serrano, D. R.; Lalatsa, A.; Dea-Ayuela, M. A. Engineering Synergistically Active and Bioavailable Cost-effective Medicines for Neglected Tropical Diseases; The Role of Excipients. Current topics in medicinal chemistry 2017.

54. Ioset, J.-R.; Brun, R.; Wenzler, T.; Kaiser, M.; Yardley, V. Drug Screening for Kinetoplastids Diseases: A Training Manual for Screening in Neglected Diseases; Drug for Neglected Diseases Initiative (DNDi) and Swiss Tropical Institute: 2009; pp 1-74.

55. Vexenat, J. A.; Croft, S. L.; Furtado Campos, J. H.; Miles, M. A. Failure of buparvaquone (Butalex) in the treatment of canine visceral leishmaniosis. Veterinary parasitology 1998, 77, (1), 71-3.

56. Pinho, B. R.; Sousa, C.; Oliveira, J. M. A.; Valentao, P.; Andrade, P. B., Bioactive compounds: Type, biological activities and health effects. . In Napthoquinones' biological activities and toxicological effects. , Bitterlich, A.; Fischl, S., Eds. Nova Science: New York, 2012; pp 181-218.

57. Ortiz, D.; Forquer, I.; Boitz, J.; Soysa, R.; Elya, C.; Fulwiler, A.; Nilsen, A.; Polley, T.; Riscoe, M. K.; Ullman, B.; Landfear, S. M. Targeting the Cytochrome bc1 Complex of Leishmania Parasites for Discovery of Novel Drugs. Antimicrobial agents and chemotherapy 2016, 60, (8), 4972-82.

58. Dey, R.; Meneses, C.; Salotra, P.; Kamhawi, S.; Nakhasi, H. L.; Duncan, R. Characterization of a Leishmania stage-specific mitochondrial membrane protein that enhances the activity of cytochrome c oxidase and its role in virulence. Molecular microbiology 2010, 77, (2), 399-414.

59. Van Hellemond, J. J.; Tielens, A. G. Inhibition of the respiratory chain results in a reversible metabolic arrest in Leishmania promastigotes. Molecular and biochemical parasitology 1997, 85, (1), 135-8.

60. McHardy, N.; Wekesa, L. S.; Hudson, A. T.; Randall, A. W. Antitheilerial activity of BW720C (buparvaquone): a comparison with parvaquone. Research in veterinary science 1985, 39, (1), 29-33.

61. Venkatesh, G.; Majid, M. I.; Ramanathan, S.; Mansor, S. M.; Nair, N. K.; Croft, S. L.; Navaratnam, V. Optimization and validation of RP-HPLC-UV method with solid-phase extraction for determination of buparvaquone in human and rabbit plasma: application to pharmacokinetic study. Biomedical chromatography : BMC 2008, 22, (5), 535-41.

62. Venkatesh, G.; Ramanathan, S.; Mansor, S. M.; Nair, N. K.; Sattar, M. A.; Croft, S. L.; Navaratnam, V. Development and validation of RP-HPLC-UV method for simultaneous determination of buparvaquone, atenolol, propranolol, quinidine and verapamil: a tool for the standardization of rat in situ intestinal permeability studies. Journal of pharmaceutical and biomedical analysis 2007, 43, (4), 1546-51.

63. Tan, A.; Rao, S.; Prestidge, C. A. Transforming lipid-based oral drug delivery systems into solid dosage forms: an overview of solid carriers, physicochemical properties, and biopharmaceutical performance. Pharmaceutical research 2013, 30, (12), 2993-3017.

64. Dunnhaupt, S.; Kammona, O.; Waldner, C.; Kiparissides, C.; Bernkop-Schnurch, A. Nano-carrier systems: Strategies to overcome the mucus gel barrier. European journal of pharmaceutics and biopharmaceutics : official journal of Arbeitsgemeinschaft fur Pharmazeutische Verfahrenstechnik e.V 2015, 96, 447-53.

65. Persson, E. M.; Gustafsson, A. S.; Carlsson, A. S.; Nilsson, R. G.; Knutson, L.; Forsell, P.; Hanisch, G.; Lennernas, H.; Abrahamsson, B. The effects of food on the dissolution of 
poorly soluble drugs in human and in model small intestinal fluids. Pharmaceutical research 2005, 22, (12), 2141-51.

66. Larsen, A. T.; Sassene, P.; Mullertz, A. In vitro lipolysis models as a tool for the characterization of oral lipid and surfactant based drug delivery systems. International journal of pharmaceutics 2011, 417, (1-2), 245-55.

67. McClements, D. J.; Xiao, H. Potential biological fate of ingested nanoemulsions: influence of particle characteristics. Food \& function 2012, 3, (3), 202-20.

68. Hauss, D. J.; Fogal, S. E.; Ficorilli, J. V.; Price, C. A.; Roy, T.; Jayaraj, A. A.; Keirns, J. J. Lipid-based delivery systems for improving the bioavailability and lymphatic transport of a poorly water-soluble LTB4 inhibitor. Journal of pharmaceutical sciences 1998, 87, (2), 1649.

69. Lalatsa, A.; Barbu, E. Carbohydrate Nanoparticles for Brain Delivery. International review of neurobiology 2016, 130, 115-53.

70. Lalatsa, A.; Schatzlein, A. G.; Uchegbu, I. F. Strategies to deliver peptide drugs to the brain. Molecular pharmaceutics 2014, 11, (4), 1081-93.

71. Augsburger, J.; Larry, L. Disintegrating agents in hard gelatin capsules. Part II: Swelling efficiency. . Drug development and industrial pharmacy 1988, 14, (9), 1235-45.

72. Visavarungroj, N.; Remon, J. P. Crosslinked starch as a disintegrant agent. . International journal of pharmaceutics 1990, 1990, (62), 2-3.

73. Craig, D. Q. The mechanisms of drug release from solid dispersions in water-soluble polymers. International journal of pharmaceutics 2002, 231, (2), 131-44.

74. Mukkavilli, R. Investigation of selected drug metabolizing cytochrome p450 enzymes and drug transporters using in vitro and in vivo animal models for drug disposition. Manipal University, Karnataka, India, 2015.

75. Sherman, I. W., Chapter 15. New Medicines Old Problems. In Malaria Genome Projects, The promise, progress and prospects, 1st ed.; Imperial College Press: Singapore, 2012; p 292.

76. Colakoglu, M.; Fidan Yaylali, G.; Yalcin Colakoglu, N.; Yilmaz, M. Successful treatment of visceral leishmaniasis with fluconazole and allopurinol in a patient with renal failure. Scandinavian journal of infectious diseases 2006, 38, (3), 208-10.

77. Rybniker, J.; Goede, V.; Mertens, J.; Ortmann, M.; Kulas, W.; Kochanek, M.; Benzing, T.; Arribas, J. R.; Fatkenheuer, G. Treatment of visceral leishmaniasis with intravenous pentamidine and oral fluconazole in an HIV-positive patient with chronic renal failure--a case report and brief review of the literature. International journal of infectious diseases : IJID : official publication of the International Society for Infectious Diseases 2010, 14, (6), e522-5. 